\title{
ON THE SPECTRUM OF THE LAPLACE OPERATOR ON THE INFINITE DIRICHLET LADDER
}

\author{
S. A. NAZAROV
}

\begin{abstract}
The spectrum of the Dirichlet problem is studied in the case of a periodic infinite planar domain having the form of an accommodation ladder: two parallel strips-uprights of thickness $h>0$ are linked by treads of the same thickness. It is shown that, for $h$ small, a gap is always opened between the second and third segments of the essential spectrum of the problem operator. The gap between the first and second segments is also discussed: its presence and characteristics depend on the distance between the uprights. It is shown that variation of the thickness of finitely many treads leads to the arising of any prescribed number of discrete spectrum points below the essential spectrum as well as inside the open gap.
\end{abstract}

\section{$\S 1$. Problem SETting}

Let $\Gamma$ be the union of the lines $\left\{x=\left(x_{1}, x_{2}\right) \in \mathbb{R}^{2}: x_{1}= \pm l / 2, x_{2} \in \mathbb{R}\right\}$ and the infinite collection of segments $\gamma_{p}=\left\{x:\left|x_{1}\right|<l / 2, x_{2}=p\right\}$ joining these lines; here $p \in \mathbb{Z}=\{0, \pm 1, \pm 2, \ldots\}$ and $l$ is a fixed positive parameter. We introduce the domain

$$
\Omega^{h}=\{x: \operatorname{dist}(x, \Gamma)<h / 2\},
$$

which has the form of an accommodation ladder (see Figure 1) with thin, of thickness $h \ll 1$, uprights and treads. Consider the Dirichlet spectral problem for the Laplace operator

$$
-\Delta_{x} u^{h}(x)=\lambda^{h} u^{h}(x), \quad x \in \Omega^{h}, \quad u^{h}(x)=0, \quad x \in \partial \Omega^{h} ;
$$

the variation form [1] of this problem looks like this:

$$
\left(\nabla_{x} u^{h}, \nabla_{x} v^{h}\right)_{\Omega^{h}}=\lambda^{h}\left(u^{h}, v^{h}\right)_{\Omega^{h}}, \quad v^{h} \in \stackrel{\circ}{H}^{1}\left(\Omega^{h}\right) .
$$

Here $\Delta_{x}=\nabla_{x} \cdot \nabla_{x}$ is the Laplacian, $\nabla_{x}=\left(\partial_{1}, \partial_{2}\right)$ is the gradient operator, the dot denotes the scalar product on $\mathbb{R}^{2}$, and $\partial_{k}=\partial / \partial x_{k}, k=1,2$. Also, $\lambda^{h}$ is the spectral parameter, $(,)_{\Omega^{h}}$ is the scalar product in the Lebesgue space $L_{2}\left(\Omega^{h}\right)$, and $\stackrel{\circ}{H}^{1}\left(\Omega^{h}\right)$ is the space of functions of the Sobolev class $H^{1}\left(\Omega^{h}\right)$ that vanish of the boundary $\partial \Omega^{h}$.

In accordance with [2, §10.1], the variational problem (1.3) gives rise to an unbounded, positive definite, selfadjoint operator $A^{h}$ in the Hilbert space $L_{2}\left(\Omega^{h}\right)$, with domain $\mathcal{D}\left(A^{h}\right) \subset \stackrel{\circ}{H}^{1}\left(\Omega^{h}\right)$. Since the inclusion $\stackrel{\circ}{H}^{1}\left(\Omega^{h}\right) \subset L_{2}\left(\Omega^{h}\right)$ is noncompact (because $\Omega^{h}$ is unbounded), the spectrum $\sigma\left(A^{h}\right)$ of $A^{h}$ has a nonempty essential component $\sigma_{e}\left(A^{h}\right)$ (see Theorem 10.1.5 in [2]) (in fact, $\sigma\left(A^{h}\right)=\sigma_{e}\left(A^{h}\right)$ ).

The Gelfand transformation [3]

$$
u^{h}(x) \mapsto U^{h}(x ; \eta)=\frac{1}{\sqrt{2 \pi}} \sum_{j \in \mathbb{Z}} e^{i \eta j} u^{h}\left(x_{1}, x_{2}+j\right)
$$

2010 Mathematics Subject Classification. Primary 35J05.

Key words and phrases. Periodic junction of thin domains, essential spectrum, Dirichlet problem, gaps, discrete spectrum.

Supported by RFBR (grant no. 09-01-00759). 


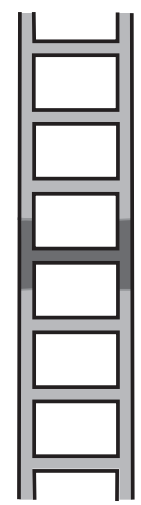

Figure 1. A planar accommodation ladder.

(the properties of it can be found, e.g., in the surveys [4, 5] and books [6, 7]) takes problem (1.2) to the following boundary-value problem with quasiperiodicity conditions:

$$
\begin{aligned}
& -\Delta_{x} U^{h}(x ; \eta)=\Lambda^{h} U^{h}(x ; \eta), \quad x \in \omega^{h}, \\
& U^{h}(x ; \eta)=0, \quad x \in \partial \omega^{h}, \quad\left|x_{2}\right|<1 / 2, \\
& U^{h}\left(x_{1},-\frac{1}{2} ; \eta\right)=e^{i \eta} U^{h}\left(x_{1}, \frac{1}{2} ; \eta\right), \\
& \frac{\partial U^{h}}{\partial x_{2}}\left(x_{1},-\frac{1}{2} ; \eta\right)=e^{i \eta} \frac{\partial U^{h}}{\partial x_{2}}\left(x_{1}, \frac{1}{2} ; \eta\right), \quad\left|x_{1}\right| \in\left(\frac{l}{2}-\frac{h}{2}, \frac{l}{2}+\frac{h}{2}\right),
\end{aligned}
$$

on an $H$-like periodicity cell (deeply tinted in Figure 1)

$$
\omega^{h}=\varpi_{0}^{h} \cup \varpi_{+}^{h} \cup \varpi_{-}^{h},
$$

where

$$
\begin{aligned}
\varpi_{0}^{h} & =\left\{x:\left|x_{1}\right|<(l-h) / 2,\left|x_{2}\right|<h / 2\right\}, \\
\varpi_{ \pm}^{h} & =\left\{x:\left|x_{1} \pm l / 2\right|<h / 2,\left|x_{2}\right|<1 / 2\right\} .
\end{aligned}
$$

Problem (1.5)-(1.7) is formally selfadjoint for any real value of the dual variable $\eta$ of the Gelfand transformation (1.4), in particular, for $\eta \in[0,2 \pi)$. This problem has discrete spectrum forming a monotone increasing positive sequence

$$
\Lambda_{1}^{h}(\eta) \leq \Lambda_{2}^{h}(\eta) \leq \ldots \leq \Lambda_{p}^{h}(\eta) \leq \ldots,
$$

where the eigenvalues are given with their multiplicities. The functions

$$
\mathbb{R} \ni \eta \mapsto \Lambda_{p}^{h}(\eta)
$$

turn out to be $2 \pi$-periodic (the change $\eta \mapsto \eta \pm 2 \pi$ does not affect the quasiperiodicity conditions (1.7)) and continuous (see [8, Chapter 9]); hence, the sets

$$
\Upsilon_{p}^{h}=\left\{\Lambda \in \mathbb{R}_{+}: \Lambda=\Lambda_{p}^{h}(\eta) \text { for some } \eta \in[0,2 \pi)\right\}
$$

are connected closed segments. It is well known (see, e.g., [4, 5] and [6, 7]) that the spectrum of the operator $A^{h}$ has the form

$$
\sigma\left(A^{h}\right)=\bigcup_{p \in \mathbb{N}} \Upsilon_{p}^{h}
$$

where $\mathbb{N}=\{1,2,3, \ldots\}$ is the set of positive integers.

The segment structure of the spectrum (1.12) allows for the arising of gaps, i.e., intervals on the real axis that are free of the spectrum of $A^{h}$, but have ends in the 
essential spectrum of $A^{h}$. Our first goal in this paper is to verify that if $h>0$ is small, then the gap $G_{23}^{h}$ between the segments $\Upsilon_{2}^{h}$ and $\Upsilon_{3}^{h}$ always exists (see Theorem 4.1). Also, in Theorem 6.1 we check that if $l<1$, then the gap $G_{12}^{h}$ is open, and that if $l \in(1,2)$, then this gap is closed. The cases where $l=1$ and $l \geq 2$ remain unexplored (for different reasons; see $\S 6$ ).

The spectrum (1.12) is essential, but if none of the segments (1.11) degenerates to a point, then the kernel of the operator of the Dirichlet problem for the Helmholtz operator $-\Delta_{x}-\lambda$ in the periodic domain $\Omega^{h}$ is finite-dimensional for all $\lambda$ (see [9] and also [6, §3.4]), and the spectrum (1.12) is continuous. In the case where $\Upsilon_{p}^{h}=\left\{\Lambda_{p}^{h}\right\}$, i.e., $\Lambda_{p}^{h}(\eta)=\Lambda_{p}^{h}$ for $\eta \in[0,2 \pi)$, the operator $A^{h}$ acquires an eigenvalue of infinite multiplicity. In any case, the discrete spectrum of $A^{h}$ is empty, but it may arise for the operator $A_{\natural}^{h}$ of problem (1.2) in a domain $\Omega_{\natural}^{h}$ obtained by perturbation of $\Omega^{h}$ via changing the thickness of one or several treads of the ladder,

$$
s_{p}^{h}=\left\{x:\left|x_{1}\right|<l / 2,\left|x_{2}-p\right|<h / 2\right\}, \quad p \in \mathbb{Z} .
$$

Our second goal in this paper is to show that such a perturbation of the domain may lead to the arising of any prescribed number of discrete spectrum points inside a gap, as well as below the essential spectrum (1.12) of the operator $A_{\natural}^{h}$.

We outline the organization of the paper. In $\S \S 2$ and 3 , we study the spectral Dirichlet problem for the Laplace operator on the union $\Xi^{H}$ of a strip of unit width and an orthogonal half-strip of width $H>0$. The solutions of this problem are used to describe the boundary layer phenomenon in the zones where uprights and treads meet (see Figure 2). In $\S 2$ we check that if $H<H_{*}\left(H_{*} \in(1,2]\right.$ is some threshold), then the operator of the problem in the domain $\Xi^{H}$ has a unique eigenvalue $\mu_{H}$ below the continuous spectrum $\left[\pi^{2} \min \left\{1, H^{-2}\right\}, \infty\right)$; also, we study the properties of the function $H \mapsto \mu_{H}$. We mention the papers [10, 11, 12, 13, 14, 15], where the spectra of broken, bent, and branching waveguides were investigated. In $\S 3$ we list all solutions of the homogeneous problem in $\Xi^{1} \ni \xi$ that have at most exponential growth $O\left(\exp \left(|\xi| \sqrt{\pi^{2}-\mu_{1}}\right)\right.$ at infinity. The boundary layer mentioned above is built from these solutions, together with the eigenfunction $w_{1} \in \dot{H}^{1}\left(\Xi^{1}\right)$ corresponding to the eigenvalue $\mu_{1}$.

In $\S 4$ we employ the maximin principle (see, e.g., [2, §10.2]) and some inequalities for functions $U^{h} \in H^{1}\left(\omega^{h}\right)$ that satisfy the Dirichlet condition (1.6) to establish the existence of a gap $G_{23}^{h}$ of length $O\left(h^{-2}\left(\pi^{2}-\mu_{1}\right)\right)$. For waveguides with other periodicity cells, the gaps in their spectra were studied in [16, 17, 18, 19].

The question about the gap $G_{12}^{h}$ is answered with the help of the asymptotic formulas for the eigenvalues $\Lambda_{1}^{h}(\eta)$ and $\Lambda_{2}^{h}(\eta)$ of problem (1.5)-(1.7), which is constructed formally in $\S 5$ and is justified in $\S 7$. This gap is always open if $l \in(0,1)$, but is closed if $l \in(1,2)$ (see $\S 6)$. The information obtained in the present paper does not suffice to study the cases of $l=1$ and $l \geq 2$. To identify the gap for $l=1$, one should find and compare the coefficients in the expansion at infinity of the eigenfunction $w_{1}$ of the boundary layer problem (see Theorem 2.1), while for $l \geq 2$ the location of the segments $\Upsilon_{1}^{h}$ and $\Upsilon_{2}^{h}$ is determined by the lower asymptotic terms, which are not constructed in this paper.

After localizing the segments $\Upsilon_{1}^{h}$ and $\Upsilon_{2}^{h}$ (see Theorem 6.1), in $\S 8$ we check that discrete spectrum points for the problem in the perturbed domain $\Omega_{\natural}^{h}$ can be found below the essential spectrum threshold (some treads become thicker) and in the gap $G_{23}^{h}$ (some treads become thinner).

In the paper, we only deal with two-dimensional problems, but all conclusions remain valid, e.g., for the Dirichlet problem in the domain $\Omega^{h} \times(-h / 2, h / 2) \subset \mathbb{R}^{3}$ (see Figure 3), because the form of a ladder made of rods with square cross-section admits separation of variables. Therefore, the part of the spectrum of the three-dimensional problem located 


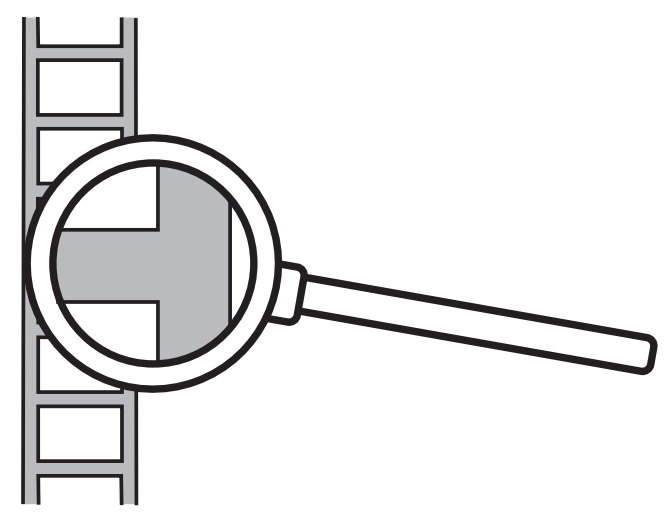

Figure 2. Coordinate dilation for the study of the boundary layer phenomenon.

on the segment $\left[0,2 h^{-2} \pi^{2}\right]$ is obtained by shifting the spectrum of problem (1.2) in the planar domain by $h^{-2} \pi^{2}$. If the ladder is composed of rods with circular cross-section, then it is not so easy to come to similar conclusions. In [14] it was shown that if a domain $\Xi$ is formed by joining an infinite circular cylinder and a perpendicular halfcylinder, then the Dirichlet problem in $\Xi$ has nonempty discrete spectrum, but the full spectrum multiplicity remained unknown. If some eigenvalues are present, then some gaps can be shown to arise, but the answer remains nonclear, and the author viewed this generalization as negligible.

\section{§2. Exponentially decaying solutions of the Dirichlet Problem IN A T-JOINT WAVEGUIDE}

Consider the Dirichlet problem

$$
-\Delta_{\xi} w(\xi)=\mu w(\xi), \quad \xi \in \Xi^{H}, \quad w(\xi)=0, \quad \xi \in \partial \Xi^{H},
$$

in a domain $\Xi^{H}$ having the form of a T-joint waveguide (see Figure 4; compare also with Figure 2). This domain is obtained by uniting three half-strips

$$
\begin{aligned}
& \Pi_{ \pm}^{H}=\left\{\xi=\left(\xi_{1}, \xi_{2}\right):\left|\xi_{1}\right|<1 / 2, \pm \xi_{2}>H / 2\right\} \\
& \Pi_{0}^{H}=\left\{\xi: \xi_{1}<1 / 2,\left|\xi_{2}\right|<H / 2\right\}
\end{aligned}
$$

and the rectangle $Q^{H}$ (deeply tinted in Figure 4),

$$
Q^{H}=\left\{\xi:-1 / 2 \leq \xi_{1}<1 / 2,-H / 2 \leq \xi_{2} \leq H / 2\right\}
$$

The variational statement of this problem looks like this:

$$
\left(\nabla_{\xi} w, \nabla_{\xi} v\right)_{\Xi^{H}}=\mu(w, v)_{\Xi^{H}}, \quad v \in \stackrel{\circ}{H}^{1}\left(\Xi^{H}\right)
$$

(cf. the integral identity (1.3)). The corresponding (see [2, §10.1]) positive selfadjoint operator $B^{H}$ in the space $L_{2}\left(\Xi^{H}\right)$ has a continuous spectrum

$$
\sigma_{c}\left(B^{h}\right)=\left[\tau_{\dagger}^{H},+\infty\right), \quad \tau_{\dagger}^{H}=\pi^{2} \min \left\{1, H^{-2}\right\} .
$$

The following assertion was verified in [15].

Theorem 2.1. There exists a critical width $H_{*} \in(1,2]$ such that $\sigma_{d}\left(B^{H}\right)=\varnothing$ if $H \geq$ $H_{*}$, but if $H \in\left(0, H_{*}\right)$, then the discrete spectrum consists of a unique (simple) eigenvalue $\mu_{H} \in\left(0, \tau_{\dagger}^{H}\right)$. 


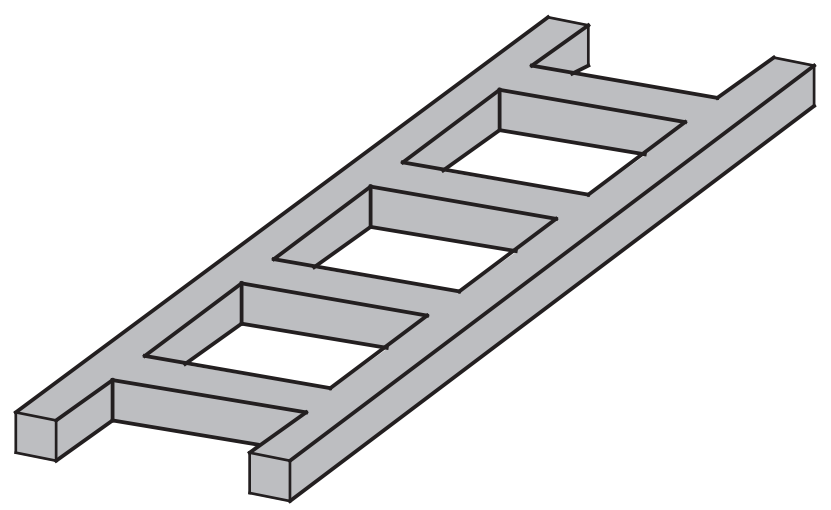

Figure 3. A spatial accommodation ladder.

In the present paper, we do not employ this theorem in its full strength. For the reader's convenience, we only verify it to the extent needed for what follows.

First, let $H \geq 1$, i.e., $\tau_{\dagger}^{H}=\pi^{2}$ (see (2.5) $)$. Suppose that the discrete spectrum of problem (2.4) includes two eigenvalues $\mu_{H}^{(1)}$ and $\mu_{H}^{(2)}$. By the maximum principle (see [2, Theorem 10.2.2]), we have

$$
\mu_{H}^{(k)}=\max _{F_{k}} \inf _{v \in F_{k} \backslash\{0\}} \frac{\left\|\nabla_{x} w ; L_{2}\left(\Xi^{H}\right)\right\|^{2}}{\left\|w ; L_{2}\left(\Xi^{H}\right)\right\|^{2}} .
$$

Here, $F_{k}$ is an arbitrary subspace of $\stackrel{\circ}{H}^{1}\left(\Xi^{H}\right)$ of codimension $k-1$, in particular, $F_{1}=$ $\stackrel{\circ}{H}^{1}\left(\Xi^{H}\right)$, i.e., for $k=1$ there is no need to calculate the maximum in (2.6).

Consider the following auxiliary spectral mixed boundary-value problem in the rectangle (2.3):

$$
\begin{aligned}
& -\Delta_{\xi} W(\xi)=M W(\xi), \quad x \in Q^{H}, \quad \partial_{2} W\left(\xi_{1}, \pm H / 2\right)=0, \quad\left|\xi_{1}\right|<1 / 2, \\
& W\left(1 / 2, \xi_{2}\right)=0, \quad \partial_{2} W\left(-1 / 2, \xi_{2}\right)=0, \quad\left|\xi_{2}\right|<1 / 2 .
\end{aligned}
$$

Clearly, $M_{1}=\pi^{2} / 4$ is its first eigenvalue, and $W_{1}(\xi)=\sin \left(\frac{\pi}{2}\left(\xi_{1}-\frac{1}{2}\right)\right)$ is the corresponding eigenfunction. The second eigenvalue looks like this:

$$
M_{2}=\pi^{2} \min \left\{\frac{9}{4}, \frac{1}{4}+\frac{1}{H^{2}}\right\}>\pi^{2} .
$$

Consequently, for any function $v \in H^{1}\left(Q^{H}\right)$ that vanishes for $\xi_{1}=1 / 2$ and satisfies the orthogonality conditions

$$
\int_{Q^{H}} w(\xi) \sin \left(\frac{\pi}{2}\left(\xi_{1}-\frac{1}{2}\right)\right) d \xi=0,
$$

we have

$$
\left\|\nabla_{\xi} w ; L_{2}\left(Q^{H}\right)\right\|^{2} \geq M_{2}\left\|w ; L_{2}\left(Q^{H}\right)\right\|^{2} \geq \pi^{2}\left\|w ; L_{2}\left(Q^{H}\right)\right\|^{2} .
$$

To (2.9) we add the one-dimensional Friedrichs inequalities

$$
\begin{aligned}
& \int_{-1 / 2}^{1 / 2}\left|\partial_{1} w\left(\xi_{1}, \xi_{2}\right)\right|^{2} d \xi_{1} \geq \pi^{2} \int_{-1 / 2}^{1 / 2}\left|w\left(\xi_{1}, \xi_{2}\right)\right|^{2} d \xi_{1} \\
& \int_{-H / 2}^{H / 2}\left|\partial_{2} w\left(\xi_{1}, \xi_{2}\right)\right|^{2} d \xi_{2} \geq \frac{\pi^{2}}{H^{2}} \int_{-H / 2}^{H / 2}\left|w\left(\xi_{1}, \xi_{2}\right)\right|^{2} d \xi_{2} \geq \pi^{2} \int_{-H / 2}^{H / 2}\left|w\left(\xi_{1}, \xi_{2}\right)\right|^{2} d \xi_{2}
\end{aligned}
$$




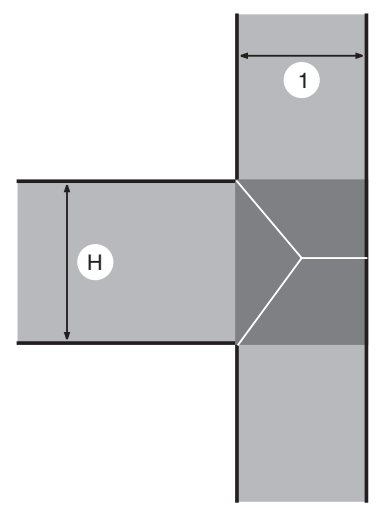

Figure 4. Plane T-joint waveguide.

integrated over $\xi_{1} \in(-\infty,-H / 2) \cup(H / 2,+\infty)$ and $\xi_{2} \in(-\infty,-1 / 2)$, respectively. This yields the estimate

$$
\left\|\nabla_{\xi} w ; L_{2}\left(\Xi^{H}\right)\right\|^{2} \geq \pi^{2}\left\|w ; L_{2}\left(\Xi^{H}\right)\right\|^{2}
$$

for any function $v$ in the subspace

$$
F_{\perp}=\left\{v \in \stackrel{\circ}{H}^{1}\left(\Xi^{H}\right): w \text { satisfies (2.8) }\right\}, \quad \operatorname{codim} F_{\perp}=1 .
$$

Using (2.6) and (2.11), we deduce the inequality

$$
\mu_{2}^{H} \geq \inf _{w \in F_{\perp} \backslash\{0\}} \frac{\left\|\nabla_{\xi} w ; L_{2}\left(\Xi^{H}\right)\right\|^{2}}{\left\|w ; L_{2}\left(\Xi^{H}\right)\right\|^{2}} \geq \pi^{2},
$$

which contradicts the assumption $\mu_{2}^{H} \in \sigma_{d}\left(B^{H}\right) \subset\left(0, \pi^{2}\right)$. Consequently, the full multiplicity of the discrete spectrum is at most one.

Now we suppose that $H \leq 1$ as before, but $\sigma_{d}\left(B^{H}\right)=\varnothing$. Then $\pi^{2}$ is the lower bound of the spectrum $\sigma\left(B^{H}\right)$ and, by [2, §10.2], inequality (2.11) is valid for all $w \in \stackrel{\circ}{H}^{1}\left(\Pi^{H}\right)$. We introduce a test function $w^{\varepsilon}$ depending on a small parameter $\varepsilon>0$ and defined on the half-strips (2.2) by the formula

$$
w^{\varepsilon}(x)= \begin{cases}\cos \left(\pi \xi_{1}\right) \exp \left(\mp \varepsilon\left(\xi_{2} \pm H / 2\right)\right), & \xi \in \Pi_{ \pm}^{H} \\ \cos \left(H^{-1} \pi \xi_{1}\right) \exp \left(\mp\left(\xi_{1}+1 / 2\right)\left(\varepsilon+\pi\left(H^{-2}-1\right)^{1 / 2}\right),\right. & \xi \in \Pi_{0}^{H}\end{cases}
$$

From the origin $\xi=0$, we draw segments to the points $(1 / 2,0)$ and $(-1 / 2, \pm H / 2)$, thus splitting the rectangle $Q^{H}$ into two rectangular trapeziums $\mathcal{T}_{ \pm}^{H}$ and an isosceles triangle $\mathcal{T}_{0}^{H}$ (see Figure 4), inside which we put

$$
w^{\varepsilon}(x)= \begin{cases}\cos \left(\pi \xi_{1}\right), & \xi \in \mathcal{T}_{ \pm}^{H} \\ \cos \left(H^{-1} \pi \xi_{1}\right), & \xi \in \mathcal{T}_{0}^{H}\end{cases}
$$

The function $w^{\varepsilon}$ decays exponentially at infinity and is piecewise smooth. It is easily seen that the definitions (2.13) agree on the separating lines in the rectangle, i.e., $w^{\varepsilon}$ is a continuous function. Thus, $w^{\varepsilon} \in \stackrel{\circ}{H}^{1}\left(\Xi^{H}\right)$. 
We plug (2.12) and (2.13) in (2.11) and calculate. Since

$$
\begin{aligned}
I\left(\Pi_{ \pm}^{H}\right) & =\left\|\nabla_{\xi} w^{\varepsilon} ; L_{2}\left(\Pi_{ \pm}\right)\right\|^{2}-\pi^{2}\left\|w^{\varepsilon} ; L_{2}\left(\Pi_{ \pm}\right)\right\|^{2} \\
I\left(\Pi_{0}^{H}\right) & =\pi^{2} \int_{-H / 2}^{H / 2} \int_{-1 / 2}^{1 / 2}\left(\frac{1}{H^{2}}\left(\sin \frac{\pi}{H} \xi_{2}\right)^{2}+\left(\frac{1}{H^{2}}-2\right)\left(\cos \frac{\pi}{H} \xi_{2}\right)^{2}\right) \\
& \times e^{2\left(\xi_{1}+1 / 2\right)\left(\varepsilon+\pi\left(H^{-2}-1\right)^{1 / 2}\right)} d \xi_{1} d \xi_{2}=\frac{\pi}{2}\left(1-h^{2}\right)^{1 / 2}+O(\varepsilon), \\
I\left(T_{ \pm}^{H}\right) & =-\frac{H}{4}, \quad I\left(T_{0}^{H}\right)=-\frac{\pi^{2}}{16}\left(1-H^{2}\right)-\frac{1}{4}\left(1+H^{2}\right),
\end{aligned}
$$

estimate (2.11) takes the form

$$
-1+\Phi(H) \geq c \varepsilon
$$

where $c$ is a constant and $\Phi$ is a continuous function on $(0,1]$ with $\Phi(1)=0$. Consequently, we can find $\varepsilon_{0}>0$ and $\delta_{-}^{H}>0$ such that if $\varepsilon \in\left(0, \varepsilon_{0}\right)$ and $H \in\left(1-\delta_{-}^{H}, 1\right]$, then (2.11) fails for $w^{\varepsilon}$, so that $\sigma_{d}\left(B^{H}\right) \neq \varnothing$.

Thus, we see that if $H \in\left(1-\delta_{-}^{H}, 1\right]$, then the discrete spectrum of $B^{H}$ consists of a unique point $\mu_{H} \in\left(0, \pi^{2}\right)$. In the case where $H \geq 1$, we contract the coordinates $\xi \mapsto H^{-1} \xi$ and repeat the above calculations with clear modifications. As a result, we find a quantity $\delta_{+}^{H} \in[0,1)$ for which $\sigma_{d}\left(B^{H}\right)=\left\{\mu_{H}\right\}$ if $H \in\left[1,1+\delta_{+}^{H}\right)$. This proves the claims of Theorem 2.1 that are needed in what follows.

Theorem 2.2. The functions $\left(0, H_{*}\right) \ni H \mapsto \mu_{H}$ and $\left(0, H_{*}\right) \ni H \mapsto H^{2} \mu_{H}$ are strictly monotone increasing and decreasing, respectively.

Proof. We apply identity (2.6) with $k=1$ (the Rayleigh formula). For $\Delta H>0$ we have $\Xi^{H+\Delta H} \supset \Xi^{H}$, whence

$$
\begin{aligned}
\mu_{H+\Delta H} & =\inf _{w \in \dot{H}^{1}\left(\Xi^{H+\Delta H}\right) \backslash\{0\}} \frac{\left\|\nabla_{x} w ; L_{2}\left(\Xi^{H+\Delta H}\right)\right\|^{2}}{\left\|w ; L_{2}\left(\Xi^{H+\Delta H}\right)\right\|^{2}} \\
& <\frac{\left\|\nabla_{x} w_{H} ; L_{2}\left(\Xi^{H+\Delta H}\right)\right\|^{2}}{\left\|w_{H} ; L_{2}\left(\Xi^{H+\Delta H}\right)\right\|^{2}}=\frac{\left\|\nabla_{x} w_{H} ; L_{2}\left(\Xi^{H}\right)\right\|^{2}}{\left\|w_{H} ; L_{2}\left(\Xi^{H}\right)\right\|^{2}}=\mu_{H} .
\end{aligned}
$$

Here $w_{H} \in \stackrel{\circ}{H}^{1}\left(\Xi^{H}\right)$ is an eigenfunction of problem (2.4) in $\Xi^{H}$ that corresponds to the eigenvalue $\mu_{H}$ and is extended by zero to the wider set $\Xi^{H+\Delta H}$. Observe that the strict inequality in (2.14) is due to two reasons. First, the eigenvalue $\mu_{H+\Delta H}$ is simple, and second, the eigenfunction $v_{H+\Delta H}$ differs from $v_{H}$ because, being a solution of the Helmholtz equation, $v_{H+\Delta H}$ cannot vanish on a set of positive measure, i.e., on $\Xi^{H} \backslash \Xi^{H+\Delta H}$ (see, e.g., 20]).

To study the properties of the function $H \mapsto \mathbf{M}_{H}=H^{2} \mu_{H}$, we make the coordinate change $\xi \mapsto H^{-1} \xi$, which takes $\Xi^{H}$ to the set

$$
\Theta^{H}=\left\{\xi: \xi_{1} \leq \frac{1}{2 H},\left|\xi_{1}\right|<\frac{1}{2}\right\} \cup\left\{\xi:\left|\xi_{1}\right|<\frac{1}{2}, \xi_{2} \in \mathbb{R}\right\} .
$$

The Dirichlet problem in $\Theta_{H}$ has the eigenvalue $\mathbf{M}_{H}$, and for $\Delta H>0$ we have $\Theta^{H+\Delta H} \subset$ $\Theta^{H}$. Applying the Rayleigh formula on the set (2.15) and repeating the above arguments, we obtain

$$
H^{2} \mu_{H}=\mathbf{M}_{H}<\frac{\left\|\nabla_{x} \mathbf{w}_{H+\Delta H} ; L_{2}\left(\Theta^{H}\right)\right\|^{2}}{\left\|\mathbf{w}_{H+\Delta H} ; L_{2}\left(\Theta^{H}\right)\right\|^{2}}=\mathbf{M}_{H+\Delta H}=(H+\Delta H)^{2} \mu_{H+\Delta H} .
$$

Here $\mathbf{w}_{H+\Delta H}(\xi)=w_{H+\Delta H}\left((H+\Delta H)^{-1} \xi\right)$ and, of course, $w_{H+\Delta H}$ is an eigenfunction of problem (2.4) in the domain $\Xi^{H+\Delta H}$ (before the coordinate change). Inequality (2.16) shows that the function $H \mapsto \mathbf{M}_{H}$ is monotone, as claimed. 


\section{§3. Exponentially Growing SOLUtions}

In this section we put $H=1$. We shall explore the solutions of problem (2.1) with the parameter $\mu=\mu_{1}$ (the eigenvalue), allowing for their possible exponential growth as $|\xi| \rightarrow \infty$. For this, we employ the theory of elliptic boundary-value problems in domains with cylindrical outlets to infinity (see the key papers 21, 22, 23, 24 and, e.g., the book 6]); we introduce the weighted Sobolev $\mathcal{W}_{\beta}^{1}\left(\Xi^{1}\right)$ with the norm

$$
\left.\left.\left\|w ; \mathcal{W}_{\beta}^{1}\left(\Xi^{1}\right)\right\|=\left(\| e^{\beta|\xi|} \nabla_{\xi} w ; L_{2}\left(\Xi^{1}\right)\right)\left\|^{2}+\right\| e^{\beta|\xi|} w ; L_{2}\left(\Xi^{1}\right)\right) \|^{2}\right)^{1 / 2}
$$

where $\beta \in \mathbb{R}$ is the weight exponent. The space $\mathcal{W}_{\beta}^{1}\left(\Xi^{1}\right)$ can be obtained by completion of the linear space $C_{c}^{1}\left(\Xi^{1}\right)$ (the infinitely differentiable functions with compact support) in the above norm, and it consists of all functions $w \in H_{l o c}^{1}\left(\overline{\Xi^{1}}\right)$ that vanish on $\partial \Xi^{1}$ and have a finite norm (3.1). Let $f \in \mathcal{W}_{-\beta}^{1}\left(\Xi^{1}\right)^{*}$ be a linear functional on the space $\mathcal{W}_{-\beta}^{1}\left(\Xi^{1}\right)$. A generalized solution of the nonhomogeneous (with right-hand side $f$ ) problem (2.1) in the class $\mathcal{W}_{\beta}^{1}\left(\Xi^{1}\right)$ is defined as a function $w$ in the above space that satisfies the integral identity

$$
\left(\nabla_{\xi} w, \nabla_{\xi} v\right)_{\Xi^{1}}-\mu_{1}(w, v)_{\Xi^{1}}=f(v), \quad v \in \mathcal{W}_{-\beta}^{1}\left(\Xi^{1}\right)
$$

see [1. Note that the left-hand side of $(3.2)$ is well defined, while $(,)_{\Xi^{1}}$ stands for the extension of the scalar product in $L_{2}\left(\Xi^{1}\right)$ up to duality between the weighted Lebesgue spaces $\mathcal{L}_{\beta}\left(\Xi^{1}\right)$ and $\mathcal{L}_{-\beta}\left(\Xi^{1}\right)$; here $\left\|v ; \mathcal{L}_{\beta}\left(\Xi^{1}\right)\right\|=\left\|e^{\beta|\xi|} v ; L_{2}\left(\Xi^{1}\right)\right\|$.

With problem (3.2) we associate a map

$$
\mathcal{B}_{\beta}: \mathcal{W}_{\beta}^{1}\left(\Xi^{1}\right) \rightarrow \mathcal{W}_{-\beta}^{1}\left(\Xi^{1}\right)^{*}
$$

such that $\mathcal{B}_{\beta} w \in \mathcal{W}_{-\beta}^{1}\left(\Xi^{1}\right)^{*}$ is the functional determined by $w \in \mathcal{W}_{\beta}^{1}\left(\Xi^{1}\right)$ as in the lefthand side of (3.2). It is known 1 (see, e.g., the introductory Chapter 2 in [6]) that the operator (3.3) is Fredholm if and only if

$$
\beta \neq \beta_{ \pm k}:= \pm \sqrt{k^{2} \pi^{2}-\mu_{1}} \quad \text { for } \quad k \in \mathbb{N} .
$$

Remark 3.1. The functions

$$
\sin \left(\pi k\left(\xi_{2}+1 / 2\right)\right) e^{ \pm \xi_{2} \sqrt{k^{2} \pi^{2}-\mu_{1}}}, \quad k= \pm 1, \pm 2, \ldots,
$$

satisfy the Helmholtz equation $\Delta_{\xi} w+\mu_{1} w=0$ in the half-strip $\Pi_{0}^{1}$ and the Dirichlet condition on the lateral sides of $\Pi_{0}^{1}$. These and similar solutions of problems in the half-strips $\Pi_{ \pm}^{1}$ give rise to the forbidden exponents $\beta_{ \pm k}$ in formula (3.4). Concerning the reasons for with the operator $\mathcal{B}_{\beta_{ \pm k}}$ loses the Fredholm property, see the source paper [21, and also the introductory Chapter 2 in the book [6], or the survey [5].

The exponent $\beta=0$ is admissible because $\mu_{1} \in\left(0, \pi^{2}\right)$. The space $\mathcal{W}_{0}^{1}\left(\Xi^{1}\right)$ coincides with the Sobolev space $\stackrel{\circ}{H}^{1}\left(\Xi^{1}\right)$. Since $\mathcal{B}_{0}$ is a selfadjoint operator and $\mu_{1}$ is its simple eigenvalue, $\mathcal{B}_{0}$ is a Fredholm operator with one-dimensional kernel and cokernel, which are spanned by the eigenfunction $w_{1}$. The theorem on asymptotics [21] (see also 6. Theorem 3.1.2]) yields the expansion

$$
w_{1}(\xi)=K_{0} X_{0}(\xi) \cos \left(\pi \xi_{2}\right) e^{m \xi_{1}}+\sum_{ \pm} K_{ \pm} X_{ \pm}(\xi) \cos \left(\pi \xi_{1}\right) e^{\mp m \xi_{2}}+\widetilde{w}_{1}(\xi)
$$

where $K_{0}$ and $K_{ \pm}$are some constants, $X_{0}$ and $X_{ \pm}$are smooth cut-off functions with supports in $\overline{\Pi_{0}^{1}}$ and $\overline{\Pi_{ \pm}^{1}}$, respectively, equal to 1 if $\max \left\{\left|\xi_{1}\right|,\left|\xi_{2}\right|\right\}>2$, and the remainder

\footnotetext{
${ }^{1}$ Usually, the theory of elliptic boundary-value problems in domains with piecewise smooth boundary deals with classically posed problems, but passage to the variational setting meets no difficulties (see [5]).
} 
term $\widetilde{w}_{1}$ lies in the space $\mathcal{W}_{\gamma}^{1}\left(\Xi^{1}\right)$ for any $\gamma<\sqrt{4 \pi^{2}-\mu_{1}}$. The last expression is the forbidden exponent $\beta_{2}$ as in (3.4), following the first such exponent

$$
m=\beta_{1}=\sqrt{\pi^{2}-\mu_{1}} .
$$

It is well known that the first eigenfunction can be fixed to be positive in $\Xi^{1}$. Also, the coefficients $K_{0}$ and $K_{ \pm}$are necessarily positive. Indeed, by the Fourier method, the solution $v_{1}$ expands on the half-strip $\left\{\xi \in \Pi_{0}^{1}: \xi_{1}<-1\right\}$ in a convergent series in the functions (3.5), $k \in \mathbb{N}$ (cf. Remark 3.1). Since

$$
\sin \left(\pi\left(\xi_{2}+1 / 2\right)\right)=\cos \left(\pi \xi_{2}\right)>0 \quad \text { for } \quad \xi_{2} \in(-1 / 2,1 / 2)
$$

we see that the coefficient $K_{0}$ is nonnegative. Suppose $K_{0}=0$; since the functions (3.5) with $k>1$ change their sign inside the half-strip $\Pi_{0}^{1}$, we can use induction to check that all the Fourier coefficients vanish, but this is impossible because no nonzero solution of the Helmholtz equation can be zero on a set of positive measure (see, e.g., 20]). In the same way, we check that $K_{ \pm}>0$. Moreover, since the domain $\Xi^{1}$ is symmetric with respect to the abscissa axis, these coefficients coincide (otherwise the eigenvalue $\mu_{1}$ would not be simple). Thus,

$$
K_{ \pm}=K>0, \quad K_{0}>0 .
$$

Let $W \in \mathcal{W}_{-\gamma}^{1}\left(\Xi^{1}\right)$ be a solution of the homogeneous problem (3.2) with

$$
\gamma \in\left(\sqrt{\pi^{2}-\mu_{1}}, \sqrt{4 \pi^{2}-\mu_{1}}\right) .
$$

This solution may grow exponentially at infinity. Using the theorem on asymptotics mentioned above, we obtain

$$
W(\xi)=C_{0} X_{0}(\xi) \cos \left(\pi \xi_{2}\right) e^{-m \xi_{1}}+\sum_{ \pm} C_{ \pm} X_{ \pm}(\xi) \cos \left(\pi \xi_{1}\right) e^{ \pm m \xi_{2}}+\widetilde{W}(\xi)
$$

where $C_{0}, C_{ \pm} \in \mathbb{R}$ are constants, and $\widetilde{W} \in \mathcal{W}_{\beta}^{1}\left(\Xi^{1}\right)$ for any $\beta<m$.

We want to find a canonical basis in the space of solutions $W$ as above. First, we note that, by formulas (3.6) and (3.8), (3.9), the eigenfunction $w_{1}$ does not lie in $\mathcal{W}_{\gamma}^{1}\left(\Xi^{1}\right)$, because the positive weight index requires too fast decay at infinity. Therefore, the operator $\mathcal{B}_{\gamma}$ is a monomorphism, so that the operator $\mathcal{B}_{-\gamma}=\mathcal{B}_{\gamma}^{*}$ adjoint to $\mathcal{B}_{\gamma}$ is an epimorphism (we have used the fact that the bilinear form on the left in (3.2) is symmetric). In other words, problem (3.2) has a solution $w \in \mathcal{W}_{-\gamma}^{1}\left(\Xi^{1}\right)$ for any right-hand side $f \in \mathcal{W}_{\gamma}^{1}\left(\Xi^{1}\right)^{*}$ (of course, this solution is nonunique). As a result, for the kernel ker $\mathcal{B}_{\gamma}$ and the cokernel coker $\mathcal{B}_{-\gamma}$ of our operators we have

$$
\operatorname{dim} \operatorname{ker} \mathcal{B}_{\gamma}=\operatorname{dim} \operatorname{coker} \mathcal{B}_{-\gamma}=0 .
$$

Also, the indices of the mutually adjoint Fredholm operators $\mathcal{B}_{\gamma}$ and $\mathcal{B}_{-\gamma}$ satisfy the relation

$$
\text { Ind } \mathcal{B}_{\gamma}=\operatorname{dim} \operatorname{ker} \mathcal{B}_{\gamma}-\operatorname{dim} \operatorname{coker} \mathcal{B}_{\gamma}=\operatorname{dim} \operatorname{coker} \mathcal{B}_{-\gamma}-\operatorname{dim} \operatorname{ker} \mathcal{B}_{-\gamma}=-\operatorname{Ind} \mathcal{B}_{-\gamma} \text {. }
$$

The theorem on the index increment (see. [6, Theorem 5.1 .4 (4)]) yields

$$
\text { Ind } \mathcal{B}_{-\gamma}=\operatorname{Ind} \mathcal{B}_{\gamma}+\varkappa N=\operatorname{Ind} \mathcal{B}_{\gamma}+6 \text {, }
$$

where $N=3$ is the number of outlets to infinity of the half-strip (2.2), and $\varkappa=2$ is the number of linearly independent exponential solutions (3.5) with the growth exponents $\pm m \in(-\gamma, \gamma)$ (in each half-strip). Comparing the above formulas, we see that

$$
\text { Ind } \mathcal{B}_{-\gamma}=\operatorname{dim} \operatorname{ker} \mathcal{B}_{-\gamma}=3 \text {. }
$$

Now we describe three linearly independent elements of ker $\mathcal{B}_{-\gamma}$. The first of them is obvious: this is the eigenfunction $w_{1} \in \stackrel{\circ}{H}^{1}\left(\Xi^{1}\right) \subset \mathcal{W}_{-\gamma}^{1}\left(\Xi^{1}\right)$ of the operator $B^{1}$ that 
corresponds to its eigenvalue $\mu_{1}$. Two other elements admit expansions as in (3.10), and at least one of the coefficients $C_{0}$ and $C_{ \pm}$in these expansions is not zero (otherwise $W \in \mathcal{W}_{0}^{1}\left(\Xi^{1}\right)$ and then the functions $W$ and $w_{1}$ are linearly dependent because the eigenvalue in question is simple). To find a formula relating the coefficients, we apply the method of [22]: we plug $W$ and $w_{1}$ in the Green formula for the truncated domain

$$
\Xi^{1}(R)=\left\{\xi \in \Xi:\left|\xi_{1}\right|<R,\left|\xi_{2}\right|<R\right\} .
$$

In this Green formula, all integrals except for those over the segments

$$
I_{ \pm}(R)=\left\{\xi: \xi_{2}= \pm R,\left|\xi_{1}\right|<1 / 2\right\}, \quad I_{0}(R)=\left\{\xi: \xi_{1}= \pm R,\left|\xi_{2}\right|<1 / 2\right\},
$$

are equal to zero, whence

$$
\begin{aligned}
\sum_{ \pm} & \pm \int_{I_{ \pm}(R)}\left(\frac{\partial W}{\partial \xi_{2}}(\xi) w_{1}(\xi)-W(\xi) \frac{\partial w_{1}}{\partial \xi_{2}}(\xi)\right) d s_{\xi} \\
& =\int_{I_{0}(R)}\left(\frac{\partial W}{\partial \xi_{1}}(\xi) w_{1}(\xi)-W(\xi) \frac{\partial w_{1}}{\partial \xi_{1}}(\xi)\right) d s_{\xi}
\end{aligned}
$$

Replacing $W$ and $w_{1}$ in the integrand by their expansions (3.10) and (3.6), we get

$$
2 m \sum_{ \pm} K_{ \pm} C_{ \pm} \int_{-1 / 2}^{1 / 2}\left(\cos \pi \xi_{1}\right)^{2} d \xi_{1}+2 m K_{0} C_{0} \int_{-1 / 2}^{1 / 2}\left(\cos \pi \xi_{2}\right)^{2} d \xi_{2}=o(1)
$$

as $R \rightarrow+\infty$. Thus, passing to the limit, we see that the coefficients occurring in (3.10) and (3.6) satisfy the relation

$$
K\left(C_{+}+C_{-}\right)+K_{0} C_{0}=0 .
$$

Since the domain $\Xi^{1}$ is symmetric, in $\operatorname{ker} \mathcal{B}_{-\gamma}$ we can find unbounded functions $W^{1}$ and $W^{2}$ such that $W^{1}$ is odd relative to $\xi_{2}$ and $W^{2}$ is even relative to $\xi_{2}$, i.e.,

$$
W^{1}\left(\xi_{1}, \xi_{2}\right)=-W^{1}\left(\xi_{1},-\xi_{2}\right), \quad W^{2}\left(\xi_{1}, \xi_{2}\right)=W^{2}\left(\xi_{1},-\xi_{2}\right) .
$$

Indeed, let $W \in \mathcal{W}_{-\gamma}^{1}\left(\Xi^{1}\right) \backslash \mathcal{W}_{\beta}^{1}\left(\Xi^{1}\right)$ be some (nontrivial) solution of the homogeneous problem (3.2). The above membership relation requires that the exponential growth and decay of $W$ as $|\xi| \rightarrow \infty$ be "not too fast". In particular, the eigenfunction $w_{1}$ does not fall into the above difference of sets $\mathcal{W}_{-\gamma}^{1}\left(\Xi^{1}\right)$ and $\mathcal{W}_{\beta}^{1}\left(\Xi^{1}\right)$ (the latter is included in the former). The functions

$$
W_{ \pm}(\xi)=\frac{1}{2}\left(W\left(\xi_{1}, \xi_{2}\right) \pm W\left(\xi_{1},-\xi_{2}\right)\right)
$$

solve the same problem and are even (plus) and odd (minus) relative to the variable $\xi_{2}$. Therefore, they can be linearly dependent only if one of them is zero. So, under the condition $W_{ \pm} \neq 0$, the required solutions are constructed. Suppose that one of the function (3.17) is zero; for definiteness, let $W_{-}=0$, i.e., $W=W_{+}$. Then the index formula (3.11) shows that there is yet another solution $W^{\prime} \in \mathcal{W}_{-\gamma}^{1}\left(\Xi^{1}\right) \backslash \mathcal{W}_{\beta}^{1}\left(\Xi^{1}\right)$ linearly independent of $w_{1}$ and $W$. Once again, we introduce the even and the odd functions

$$
W_{ \pm}^{\prime}(\xi)=\frac{1}{2}\left(W^{\prime}\left(\xi_{1}, \xi_{2}\right) \pm W^{\prime}\left(\xi_{1},-\xi_{2}\right)\right) .
$$

If $W_{-}^{\prime} \neq 0$, then the claim is proved, because $W$ and $W_{-}^{\prime}$ are linearly independent. If $W_{-}^{\prime}=0$ and $W^{\prime}=W_{+}^{\prime}$, then an appropriate linear combination $c W+c^{\prime} W^{\prime}$ admits expansion as in (3.10) with $C_{ \pm}=0$, but then the third coefficient $C_{0}$ is equal to zero, by (3.15), i.e., $c W+c^{\prime} W^{\prime} \in \mathcal{W}_{\beta}^{1}\left(\Xi^{1}\right)$ and $c W+c^{\prime} W^{\prime}=c_{1} w_{1}$ with some constant $c_{1}$. In other words, the functions $W, W^{\prime}$, and $w_{1}$ are linearly dependent, which contradicts our assumption; hence, $W_{-}^{\prime} \neq 0$, and we may take $W^{1}=W_{-}^{\prime}$ and $W^{2}=W$. 
In accordance with (3.15), after normalization, the coefficients $C^{q}$ and $C_{ \pm}^{q}$ in the representation (3.10) of the function $W^{q}$ take the form

$$
C_{ \pm}= \pm 1, C_{0}=0 \quad \text { and } \quad C_{ \pm}=1, C_{0}=-2 K_{0}^{-1} K .
$$

Thus, we have constructed the desired basis $\left\{w_{1}, W_{1}, W_{2}\right\}$ in the space of solutions of the homogeneous problems (3.2) that belong to the space $\mathcal{W}_{-\gamma}^{1}\left(\Xi^{1}\right)$ with the weight exponent (3.9), i.e., possess at most exponential growth $O\left(e^{m|\xi|}\right)$ as $|\xi| \rightarrow+\infty$.

The eigenfunction $w_{1} \in \stackrel{\circ}{H}^{1}\left(\Xi^{1}\right)$ could be normalized by the condition

$$
\left\|w_{1} ; L_{2}\left(\Xi^{1}\right)\right\|=1,
$$

but we shall not do this because, quite possibly, other normalizations can turn out to be more convenient for calculating the coefficients (3.8). Of course, all further results do not depend of the choice of a specific eigenfunction. In particular, relations (3.18) and (3.16) fix the functions $W_{1}$ and $W_{2}$ to be used in what follows uniquely.

\section{$\S 4$. EXISTENCE OF THE GAP}

The model spectral problem (1.5) - (1.7) on the periodicity cell (1.8) admits a variational setting:

$$
\left(\nabla_{x} U^{h}, \nabla_{x} V^{h}\right)_{\omega^{h}}=\Lambda^{h}(\eta)\left(U^{h}, V^{h}\right)_{\omega^{h}}, \quad V^{h} \in \mathcal{H}(\eta),
$$

where $\mathcal{H}(\eta)$ is the subspace of functions that lie in the Sobolev class $H^{1}\left(\omega^{h}\right)$ and satisfy the Dirichlet condition (1.6) and the first quasiperiodicity condition (1.7). For clear reasons, the bilinear form on the left-hand side of (4.1) is a scalar product in the Hilbert-space $\mathcal{H}(\eta)$. By [2, Theorem 10.2.2], the eigenvalues (1.10) can be found with the help of the maximin principle:

$$
\Lambda_{k}^{h}(\eta)=\max _{\mathcal{F}_{k}(\eta)} \inf _{U^{h} \in \mathcal{F}_{k}(\eta) \backslash\{0\}} \frac{\left\|\nabla_{x} U^{h} ; L_{2}\left(\omega^{h}\right)\right\|^{2}}{\left\|U^{h} ; L_{2}\left(\omega^{h}\right)\right\|^{2}},
$$

where $\mathcal{F}_{k}(\eta)$ is an arbitrary subspace in $\mathcal{H}(\eta)$ of codimension $k-1$.

We use formula (4.2) to obtain an upper estimate for the eigenvalue $\Lambda_{2}^{h}(\eta)$ and a lower estimate for $\Lambda_{3}^{h}(\eta)$. First, we observe that any subspace $\mathcal{F}_{2}(\eta)$ of codimension 1 contains a nontrivial linear combination

$$
a_{1} U_{+}^{h}+a_{2} U_{-}^{h}
$$

of the functions

$$
U_{ \pm}^{h}(x)=\chi_{ \pm}(x) w_{1}\left( \pm h^{-1}\left(x_{1} \mp l / 2\right), h^{-1} x_{2}\right) .
$$

Here, $w_{1}$ is an eigenfunction of the operator $B^{1}$ of problem (2.4) in the domain $\Xi^{1}$ that corresponds to the eigenvalue $\mu_{1} \in\left(0, \pi^{2}\right)$, and $\chi_{ \pm}$is a smooth cut-off function equal to 1 if $\left|x_{1} \mp l / 2\right|<\rho / 3$ and $\left|x_{2}\right|<\rho / 3$, and to zero of $\left|x_{1} \mp l / 2\right|>2 \rho / 3$, or $\left|x_{2}\right|>2 \rho / 3$, where $\rho=\min \{1, l\}$.

To treat the denominator and numerator of the Rayleigh fraction in (4.2), note that, since the supports of the cutoff functions $\chi_{ \pm}$are disjoint, the coordinate change $x \mapsto$ $\left.h^{-1}\left( \pm x_{1}-l / 2\right), x_{2}\right)$ yields

$$
\begin{aligned}
\left\|a_{1} U_{+}^{h}+a_{2} U_{-}^{h} ; L_{2}\left(\omega^{h}\right)\right\|^{2} & =\left(\left|a_{1}\right|^{2}+\left|a_{2}\right|^{2}\right) h^{2} \int_{\Xi^{1}}\left|w_{1}(\xi)\right|^{2} \chi_{ \pm}\left( \pm h \xi_{1}+l / 2, h \xi_{2}\right)^{2} d \xi \\
& =|a|^{2} h^{2}\left(\left\|w_{1} ; L_{2}\left(\Xi^{1}\right)\right\|^{2}+O\left(\exp \left(-\frac{2}{3} \frac{\rho}{h} m\right)\right)\right) .
\end{aligned}
$$


We have used the fact that $w_{1}(\xi)=O\left(\exp \left(-h^{-1} \rho m / 3\right)\right)$ on the support of the cut-off function $1-\chi_{ \pm}$; the factor of $h^{2}$ is due to coordinate dilation. Similarly, we have

$$
\begin{aligned}
& \left\|a_{1} \nabla_{x} U_{+}^{h}+a_{2} \nabla_{x} U_{-}^{h} ; L_{2}\left(\omega^{h}\right)\right\|^{2} \\
& \quad=|a|^{2}\left(\left\|\nabla_{\xi} w_{1} ; L_{2}\left(\Xi^{1}\right)\right\|^{2}+O\left(\exp \left(-2 h^{-1} m /(3 \rho)\right)\right)\right) .
\end{aligned}
$$

Now we use the interval identity (2.4) with $w=v=w_{1}, \mu=\mu_{1}$, and the maximin principle (4.2) to deduce that

$$
\begin{aligned}
\Lambda_{2}^{h}(\eta) & =\max _{\mathcal{F}_{k}(\eta)} \frac{\left\|a_{1} \nabla_{x} U_{+}^{h}+a_{2} \nabla_{x} U_{-}^{h} ; L_{2}\left(\omega^{h}\right)\right\|^{2}}{\left\|a_{1} U_{+}^{h}+a_{2} U_{-}^{h} ; L_{2}\left(\omega^{h}\right)\right\|^{2}} \\
& \leq \frac{1}{h^{2}} \frac{\left\|\nabla_{\xi} w_{1} ; L_{2}\left(\Xi^{1}\right)\right\|^{2}+c \exp \left(-2 h^{-1} \frac{m}{3 \rho}\right)}{\left\|w_{1} ; L_{2}\left(\Xi^{1}\right)\right\|^{2}-c \exp \left(-2 h^{-1} \frac{m}{3 \rho}\right)} \leq \frac{1}{h^{2}}\left(\mu_{1}+C \exp \left(-\frac{2}{3} \frac{\rho}{h} m\right)\right) .
\end{aligned}
$$

Consider the next eigenvalue $\Lambda_{3}^{h}(\eta)$. First, observe that the one-dimensional Friedrichs inequality (cf. (2.10) $)$ on the small interval $(-h / 2, h / 2)$ ensures the relation

$$
\left\|\nabla_{x} U^{h} ; L_{2}\left(\omega^{h} \backslash\left(Q_{-}^{h} \cup Q_{+}^{h}\right)\right)\right\|^{2} \geq \pi^{2} h^{-2}\left\|U^{h} ; L_{2}\left(\omega^{h} \backslash\left(Q_{-}^{h} \cup Q_{+}^{h}\right)\right)\right\|^{2},
$$

where $Q_{ \pm}^{h}$ is the square with side length $h$ centered at $( \pm l / 2,0)$. Under the orthogonality condition

$$
\int_{Q_{ \pm}^{h}} U^{h}(x) \sin \left(\frac{\pi}{2 h}\left(x_{1} \mp \frac{1}{2}(l+h)\right)\right) d x=0
$$

we have

$$
\left\|\nabla_{x} U^{h} ; L_{2}\left(Q_{ \pm}^{h}\right)\right\|^{2} \geq \frac{5}{4} \frac{\pi^{2}}{h^{2}}\left\|U^{h} ; L_{2}\left(Q_{ \pm}^{h}\right)\right\|^{2}
$$

(cf. formulas (2.7)-(2.9)). Adding (4.7) and (4.9), we see that any function $U^{h}$ in the subspace

$\mathcal{F}_{3}^{\perp}(\eta)=\left\{U^{h} \in \mathcal{H}(\eta)\right.$ : the two conditions in (4.8) are fulfilled $\}, \operatorname{codim} \mathcal{F}_{3}^{\perp}(\eta)=2$, satisfies the estimate

$$
\left\|\nabla_{x} U^{h} ; L_{2}\left(\omega^{h}\right)\right\|^{2} \geq \frac{\pi^{2}}{h^{2}}\left\|U^{h} ; L_{2}\left(\omega^{h}\right)\right\|^{2} .
$$

Now formula (4.2) shows that

$$
\Lambda_{3}^{h}(\eta) \geq \inf _{U^{h} \in \mathcal{F}_{3}^{\perp}(\eta) \backslash\{0\}} \frac{\left\|\nabla_{x} U^{h} ; L_{2}\left(\omega^{h}\right)\right\|^{2}}{\left\|U^{h} ; L_{2}\left(\omega^{h}\right)\right\|^{2}} \geq \frac{\pi^{2}}{h^{2}} .
$$

We summarize, stating an assertion that follows from (4.6) and (4.10).

Theorem 4.1. For small $h>0$, the essential spectrum (1.12) has a gap $G_{23}^{h}=\left(g_{2}^{h}, g_{3}^{h}\right)$ between the segments $\Upsilon_{2}^{h}$ and $\Upsilon_{3}^{h}$, and

$$
g_{3}^{h} \geq \frac{\pi^{2}}{h^{2}}>\frac{1}{h^{2}}\left(\mu_{1}+C \exp \left(-\frac{2}{3} \frac{\rho}{h} m\right)\right) \geq g_{2}^{h},
$$

i.e., the gap length is at least

$$
h^{-2}\left(\pi^{2}-\mu_{1}-C \exp \left(-2 h^{-1} m /(3 \rho)\right)\right)=O\left(h^{-2}\left(\pi^{2}-\mu_{1}\right)\right) .
$$




\section{§5. Formal asymptotics of eigenvalues of the model Problem}

In the preceding section it was checked that for any $\eta \in[0,2 \pi)$, on the interval $\left(0, h^{-2} \pi^{2}\right)$ the boundary-value problem (1.5)-(1.7) (or the variational problem (4.1)) has precisely two eigenvalues $\Lambda_{1}^{h}(\eta)$ and $\Lambda_{2}^{h}(\eta)$. We construct their asymptotics. To facilitate calculations, observe that, by the symmetry of the periodicity cell $\omega^{h}$ relative to the ordinate axis, the corresponding eigenfunctions satisfy

$$
\partial_{1} U_{1}^{h}\left(0, x_{2} ; \eta\right)=0, \quad U_{2}^{h}\left(0, x_{2} ; \eta\right)=0, \quad\left|x_{2}\right|<h / 2 .
$$

In other words, $U_{1}^{h}$ is an even function of $x_{1}$, and $U_{2}^{h}$ is an odd function of $x_{1}$. The artificial boundary conditions (5.1) order the eigenvalues: the minimum principle (see, e.g., [2, Theorem 10.1.1]) shows that

$$
\Lambda_{1}^{h}(\eta)<\Lambda_{2}^{h}(\eta)
$$

not ensuring, however, the existence of a gap between the segments $\Upsilon_{1}^{h}$ and $\Upsilon_{2}^{h}$ (because $\Lambda_{p}^{h}(\eta)$ in (1.11) depends on the parameter $\left.\eta \in[0,2 \pi)\right)$.

First, the eigenvalue asymptotics will be constructed in the case where $l=1$. For the role of the asymptotic approximation to $U_{q}^{h}$ on the thin strip $\varpi_{+}^{h}$ (see (1.9) ), we take the sum

$$
\begin{array}{r}
w_{1}\left(h^{-1}\left(x_{1}-l / 2\right), h^{-1} x_{2}\right)+e^{-m / h}\left(a_{q}(\eta) W_{1}\left(h^{-1}\left(x_{1}-l / 2\right), h^{-1} x_{2}\right)\right. \\
\left.+b_{q}(\eta) W_{2}\left(h^{-1}\left(x_{1}-l / 2\right), h^{-1} x_{2}\right)\right) .
\end{array}
$$

Here $w_{1}$ is an eigenfunction of the operator $B^{1}$ (see $\S 2$ and formulas (3.6) and (3.8)), $m$ is the exponent (3.7) corresponding to the eigenvalue $\mu_{1} \in\left(0, \pi^{2}\right)$, the $W_{j}$ are solutions of the homogeneous problem (2.1) that satisfy (3.16) and (3.10), (3.18), and the coefficients $a_{q}(\eta)$ and $b_{q}(\eta)$ are to be determined.

We substitute the sum (5.3) in the quasiperiodicity conditions (1.7) and replace the summands with their representations (3.6), (3.8), and (3.10), (3.18) as $\xi_{2}=h^{-1} x_{2} \rightarrow$ $\pm \infty$. The factor $e^{-m / h}$ in (5.3) is chosen so that all expressions be of one and the same order at $x_{2}= \pm 1 / 2$. Thus, looking only at the leading asymptotic terms, from the quasiperiodicity conditions we deduce the equations

$$
\begin{aligned}
& K-a_{q}(\eta)+b_{q}(\eta)=e^{i \eta}\left(K+a_{q}(\eta)+b_{q}(\eta)\right), \\
& K+a_{q}(\eta)-b_{q}(\eta)=e^{i \eta}\left(-K+a_{q}(\eta)+b_{q}(\eta)\right) .
\end{aligned}
$$

The identity in the first line comes from the first quasiperiodicity condition (1.7) for the function itself, and that in the second line corresponds to the quasiperiodicity condition for the derivative. Differentiation of the exponentials $e^{ \pm \xi_{1}}$ and $e^{\mp \xi_{1}}$ has led to changing the signs of the coefficients $K$ and $a_{q}, b_{q}$, and the common factor $e^{-m / h} \cos \left(\pi \xi_{2}\right)$ is omitted.

Solving the system (5.4), we get

$$
b_{q}(\eta)=\frac{K}{2}\left(e^{i \eta}+e^{i \eta}\right)=K \cos \eta, \quad a_{q}(\eta)=\frac{K}{2}\left(e^{-i \eta}-e^{i \eta}\right)=i K \sin \eta .
$$

By (3.6), (3.8) and (3.10), (3.18), for $x_{1}=0$ the expression (5.3) takes the form

$$
\cos \left(\pi x_{2} / 2\right) e^{-m /(2 h)}\left(K_{0}-2 K_{0}^{-1} K b_{q}(\eta)\right)+\ldots
$$

(recall that $l=1$ by assumption). Formula (5.6) has no free coefficients, so that we cannot satisfy the artificial Dirichlet condition (5.1) (or the Neumann condition). We modify the asymptotic Ansatz for $U_{q}^{h}(x ; \eta)$, adding yet another term

$$
e^{-m / h} \Lambda_{q}^{\prime}(\eta) W^{\prime}\left(h^{-1}\left(x_{1}-l / 2\right), h^{-1} x_{2}\right)
$$


to the sum (5.3). Simultaneously, we take the following asymptotic Ansatz for the eigenvalue:

$$
\Lambda_{q}^{h}(\eta)=h^{-2} \mu_{1}+e^{-m / h} \Lambda_{q}^{\prime}(\eta)+\ldots
$$

In (5.7), $W^{\prime}$ is the solution of the nonhomogeneous problem (2.1),

$$
-\Delta_{\xi} W^{\prime}(\xi)-\mu_{1} W^{\prime}(\xi)=w_{1}(\xi), \quad \xi \in \Xi^{1}, \quad W^{\prime}(\xi)=0, \quad \xi \in \partial \Xi^{1} .
$$

In other words, the additional summand (5.7) should compensate for the discrepancy generated by the perturbation (5.8) of the eigenvalue.

In accordance with $\S 3$, there exists a (nonunique) solution of problem (5.9) admitting the expansion (3.10) with coefficients $C_{0}^{\prime}$ and $C_{ \pm}^{\prime}$ (see Remark 7.1 below). Adding an appropriate linear combination $c_{1}^{\prime} W_{1}+c_{2}^{\prime} W_{2}$ to a particular solution and using (3.18), we can arrange that the solution decay exponentially in the half-strips $\Pi_{ \pm}^{1}$ as $\xi_{2} \rightarrow \pm \infty$, i.e., that

$$
C_{ \pm}^{\prime}=0
$$

The requirement (5.10) allows us to keep the calculations (5.4) and (5.5): indeed, since $W^{\prime}$ decays as $\xi_{2} \rightarrow \pm \infty$, the summand (5.7) for $x_{2}= \pm 1 / 2$ is an infinitely small quantity of the next order as compared to the terms of (5.3).

We compute the coefficient $C_{0}^{\prime}$ in the expansion (5.10) of $W^{\prime}$, which is uniquely fixed now by the requirement (3.10); this coefficient will be denoted by $\mathcal{K}$. We apply the method of 22 and repeat the calculations of (3.13), (3.14) with obvious modifications. Letting $R \rightarrow+\infty$ in the Green formula for the functions $W^{\prime}$ and $w_{1}$ on the domain (3.12), we obtain

$$
\begin{aligned}
\left\|w_{1} ; L_{2}\left(\Xi^{1}\right)\right\|^{2} & =\lim _{R \rightarrow \infty} \int_{\Xi^{1}(R)}\left(-w_{1}(\xi)\left(\Delta_{\xi}+\mu_{1}\right) W^{\prime}(\xi)+W^{\prime}(\xi)\left(\Delta_{\xi}+\mu_{1}\right) w_{1}(\xi)\right) d \xi \\
& =\lim _{R \rightarrow \infty} \int_{I_{0}(R)}\left(w_{1}(\xi) \frac{\partial W^{\prime}}{\partial \xi_{1}}(\xi)+W^{\prime}(\xi) \frac{\partial w_{1}}{\partial \xi_{1}}(\xi)\right) d s_{\xi}=-\mathcal{K} m K_{0}
\end{aligned}
$$

or

$$
\mathcal{K}=-m^{-1} K_{0}^{-1}\left\|w_{1} ; L_{2}\left(\Xi^{1}\right)\right\|^{2} .
$$

Now, the sum of (5.3) and (5.7) at $x_{1}=0$ is equal to

$$
\cos \left(\pi x_{2} / h\right) e^{-m /(2 h)}\left(K_{0}-2 K_{0}^{-1} K b_{2}(\eta)+\mathcal{K} \Lambda_{2}^{\prime}(\eta)\right)+\ldots,
$$

so that the Dirichlet condition in (5.1) will be fulfilled in the leading terms if

$$
\Lambda_{2}^{\prime}(\eta)=-\frac{1}{\mathcal{K}}\left(K_{0}-2 K_{0}^{-1} K b_{2}(\eta)\right)=\frac{m}{\left\|w_{1} ; L_{2}\left(\Xi^{1}\right)\right\|^{2}}\left(K_{0}^{2}-K^{2} \cos \eta\right) .
$$

Here we have used (5.5) and (5.11).

Similarly, the $x_{1}$-derivative of the same sum at $x_{1}=0$ can be written as

$$
h^{-1} m \cos \left(\pi x_{2} / h\right) e^{-m /(2 h)}\left(K_{0}+2 K_{0}^{-1} K b_{1}(\eta)-\mathcal{K} \Lambda_{2}^{\prime}(\eta)\right)+\ldots,
$$

because the factor of $K_{0}$ is the exponential $\exp \left(m h^{-1}\left(x_{1}-l / 2\right)\right)$, while the factors of $2 K_{0}^{-1} K b_{1}(\eta)$ and $\mathcal{K}$ are the exponential $\exp \left(-m h^{-1}\left(x_{1}-l / 2\right)\right)$. As a result, the artificial Neumann boundary condition (5.1) leads to the relation

$$
\Lambda_{1}^{\prime}(\eta)=-\frac{m}{\left\|w_{1} ; L_{2}\left(\Xi^{1}\right)\right\|^{2}}\left(K_{0}^{2}+K^{2} \cos \eta\right) .
$$

The error terms in the representations (5.8), refined by (5.14) and (5.13), will be estimated in the next section. It should be emphasized that, by the second formula in (3.8), for any $\eta \in[0,2 \pi)$ we have the inequality $\Lambda_{1}^{\prime}(\eta)<\Lambda_{2}^{\prime}(\eta)$, which is in agreement with the relationship (5.2) between the eigenvalues $\Lambda_{1}^{h}(\eta)$ and $\Lambda_{2}^{h}(\eta)$ of the model problem. 
Now we assume that $l \neq 1$, but preserve the asymptotic Ansatz (5.8) for eigenvalues, allowing for the dependence of $\Lambda^{\prime}(\eta)$ on the parameter $h$. The expression (5.12) will be replaced with

$$
\cos \left(\pi x_{2} / h\right)\left(e^{-m l /(2 h)} K_{0}-e^{-m / h} e^{m l /(2 h)}\left(2 K_{0}^{-1} K b_{2}(\eta)+\mathcal{K} \Lambda_{2}^{\prime}(\eta)\right)\right)+\ldots
$$

We act much as in the case of $l=1$; to satisfy the Dirichlet conditions in (5.1), we put

$$
\Lambda_{2}^{h \prime}(\eta)=\frac{m}{\left\|w_{1} ; L_{2}\left(\Xi^{1}\right)\right\|^{2}}\left(e^{-m(l-1) / h} K_{0}^{2}-K^{2} \cos \eta\right) .
$$

The Neumann conditions in (5.1) require that

$$
\Lambda_{1}^{h^{\prime}}(\eta)=-\frac{m}{\left\|w_{1} ; L_{2}\left(\Xi^{1}\right)\right\|^{2}}\left(e^{-m(l-1) / h} K_{0}^{2}+K^{2} \cos \eta\right)
$$

\section{§6. Attempts to IDENTIFy the GAP}

First, let $l=1$. In the next section, the asymptotic formulas (5.7) with the correction terms (5.14) and (5.11) will be justified, i.e., we shall estimate the discrepancies

$$
\widetilde{\Lambda}_{q}^{h}(\eta)=\Lambda_{q}^{h}(\eta)-h^{-2} \mu_{1}-e^{-m / h} \Lambda_{q}^{h \prime}(\eta), \quad q=1,2 .
$$

Thus, by formulas (1.11) and (5.14), (5.11), the segments $\Upsilon_{1}^{h}$ and $\Upsilon_{2}^{h}$ of the essential spectrum (1.12) of problem (1.2) satisfy the relations

$$
\hat{\Upsilon}_{q}^{h-} \subset \Upsilon_{q}^{h} \subset \hat{\Upsilon}_{q}^{h+}, \quad q=1,2,
$$

where

$$
\begin{aligned}
& \hat{\Upsilon}_{1}^{h \pm}=\left[h^{-2} \mu_{1}-e^{-m / h} M\left(K_{0}^{2}+K^{2}\right) \mp\left|\widetilde{\Lambda}_{1}^{h}\right|, h^{-2} \mu_{1}-e^{-m / h} M\left(K_{0}^{2}-K^{2}\right) \pm\left|\widetilde{\Lambda}_{1}^{h}\right|\right], \\
& \widehat{\Upsilon}_{2}^{h \pm}=\left[h^{-2} \mu_{1}+e^{-m / h} M\left(K_{0}^{2}-K^{2}\right) \mp\left|\widetilde{\Lambda}_{2}^{h}\right|, h^{-2} \mu_{1}+e^{-m / h} M\left(K_{0}^{2}+K^{2}\right) \pm\left|\widetilde{\Lambda}_{2}^{h}\right|\right] .
\end{aligned}
$$

Also, $M=m\left\|w_{1} ; L_{2}\left(\Xi^{1}\right)\right\|^{-2}$, and $\left|\widetilde{\Lambda}_{q}^{h}\right|$ is the upper bound for the modulus of the quantities (6.1) for $\eta \in[0,2 \pi)$. In Theorem 7.1 it will be established that $\left|\widetilde{\Lambda}_{q}^{h}\right|=$ $o\left(e^{-m(1+\delta) / h}\right.$ ) with some $\delta>0$ (recall that $l=1$ so far), i.e., formula (6.2) gives a correct impression about the location and the size of the segments in question. Unfortunately, on this basis we cannot judge whether the gap $G_{12}$ is closed or open, because we do not know the values of the coefficients $K$ and $K_{0}$, more precisely, of their ratio $K^{-1} K_{0}$. The following situations are possible. If $K>K_{0}$, then for sufficiently small $h$ the segments $\hat{\Upsilon}_{1}^{h+}$ and $\hat{\Upsilon}_{2}^{h+}$ do not intersect; hence, $\Upsilon_{1}^{h} \cap \Upsilon_{2}^{h}=\varnothing$ and the gap is open. On the contrary, if $K<K_{0}$, then the segments $\widehat{\Upsilon}_{1}^{h-}$ and $\widehat{\Upsilon}_{2}^{h-}$, included in $\Upsilon_{1}^{h}$ and $\Upsilon_{2}^{h}$ (respectively), mutually intersect, and then the gap is closed. Finally, if $K=K_{0}$, then the constructed asymptotic corrections do not suffice for getting any reliable information.

Not stating a conditional result for $l=1$, we pass to the situation where $l \neq 1$. Relations (6.2) and (6.3) remain valid, but $K_{0}^{2}$ should be replaced with $e^{-m(l-1) / h} K_{0}^{2}$ in the last term. If $l \in(0,1)$, then the extra factor $e^{-m(l-1) / h}$ is grows unboundedly as $h \rightarrow+0$; consequently, the centers of the segments $\hat{\Upsilon}_{1}^{h+}$ and $\hat{\Upsilon}_{2}^{h+}$ lie at a distance of $O\left(e^{-m l / h}\right)$, which is larger than $2 M K^{2} e^{-m / h}$. Thus, under the condition $\left|\widetilde{\Lambda}_{q}^{h}\right|=$ $o\left(e^{-m l / h}\right)$, the sets $\Upsilon_{1}^{h}$ and $\Upsilon_{2}^{h}$ are disjoint, and the gap is open. On the contrary, if $l>1$, then the centers of the segments $\hat{\Upsilon}_{1}^{h-}$ and $\hat{\Upsilon}_{2}^{h-}$ are only $O\left(e^{-m l / h}\right)$ apart, and their distance can be overlapped when the parameter $\cos \eta \in[-1,1]$ varies. Consequently, in this case there is no gap provided $\left|\widetilde{\Lambda}_{q}^{h}\right|=o\left(e^{-m / h}\right)$.

Note that formulas (5.16) and (5.17) violate the usual rules of constructing asymptotic expansions: they involve terms with different orders of smallness. Therefore, we can fully justify the asymptotic formulas in question only if $l<2$. To study the case where $l \geq 2$, 
lower asymptotic terms are needed. Some general procedures for constructing asymptotic series in long (thin) cylindrical domains are known (see [25] and also [26, §5.6]), but we have two reasons not to deal with the full series in the present paper. First, such series are very bulky, and second, they are to a great extent useless, because, in any case, we know of no sharp values of the coefficients (3.8) in the expansion (3.6) for the eigenfunction.

The next assertions will be obtained in $\S 7$ as consequences of Theorem 7.1 on the asymptotics of the eigenvalues $\Lambda_{1}^{h}(\eta)$ and $\Lambda_{2}^{h}(\eta)$.

Theorem 6.1. 1) For any $l>0$, we can find $h_{1}(l)>0$ and $C(l)>0$ such that if $h \in\left(0, h_{1}(l)\right)$, then the segments $\Upsilon_{1}^{h}$ and $\Upsilon_{2}^{h}$ of the essential spectrum (1.12) lie in the interval

$$
\left(h^{-2} \mu_{1}-C e^{-m \min \{1, l\} / h}, h^{-2} \mu_{1}-C(l) e^{-m \min \{1, l\} / h}\right) .
$$

2) If $l \in(0,1)$, then the segments $\Upsilon_{1}^{h}$ and $\Upsilon_{2}^{h}$ are disjoint provided $h \in\left(0, h_{2}(l)\right)$, where $h_{2}(l)$ is some positive number. The gap $G_{12}^{h}$ is open; its length is $e^{-m / h}\left(2 m \bar{K}_{0}^{2}+o(1)\right)$ as $h \rightarrow+0$, where $m$ is the exponent (3.7), and $\bar{K}_{0}$ is the coefficient in the expansion of the eigenfunction $\bar{w}_{1}$ of the Dirichlet problem (2.1) in the T-shape waveguide $\Xi^{1}$, normalized as in (3.19).

3) If $l \in(1,2)$, then for small $h>0$ the gap $G_{12}^{h}$ is closed: the segments $\Upsilon_{1}^{h}$ and $\Upsilon_{2}^{h}$ have points in common for $h \in\left(0, h_{3}(l)\right)$; here $h_{3}(l)>0$.

\section{$\S 7$. Justification of Asymptotics}

Consider problem (1.5)-(1.7) with the artificial Neumann or Dirichlet boundary conditions (5.1) on the segment $I_{0}^{h}=\left\{x: x_{1}=0,\left|x_{2}\right|<h / 2\right\}$, i.e., equation (1.5) will be restricted to the right half $\omega_{+}^{h}=\left\{x \in \omega^{h}: x_{1}>0\right\}$ of the periodicity cell, and the boundary conditions (1.6) and quasiperiodicity conditions (1.7) will be restricted to the corresponding part of the boundary $\partial \omega^{h}$. The operators of the problems obtained in this way will be denoted by $\mathcal{A}_{1}^{h}(\eta)$ (the Neumann condition) and $\mathcal{A}_{2}^{h}(\eta)$ (the Dirichlet condition). By estimate (4.10), problem (1.5)-(1.7) has two eigenvalues $\Lambda_{1}^{h}(\eta)$ and $\Lambda_{2}^{h}(\eta)$ on the interval $\left(0, h^{-2} \pi^{2}\right)$; these eigenvalues satisfy (5.2) and give rise to eigenfunctions that are even and odd (respectively) relative to $x_{1}$. Therefore, each of the operators $\mathcal{A}_{q}^{h}(\eta)$ has only one eigenvalue $\Lambda_{q}^{h}(\eta)$.

Also, each of these operators is positive and selfadjoint in the space $L_{2}\left(\omega_{+}^{h}\right)$, and has a purely discrete spectrum. The lemma on "near eigenvalues and near eigenvectors" (see [27], and also [2, Chapter 6]) implies the following claim.

Lemma 7.1. Given a number $\mathcal{L}_{q}^{h}(\eta)$, suppose that a function $\mathcal{U}_{q}^{h}(\cdot, \eta) \in H^{1}\left(\omega_{+}^{h}\right)$ satisfies the boundary condition (1.6), the first quasiperiodicity condition (1.7), and also the Dirichlet condition (5.1) if $q=2$. Then the segment

$$
\left[\mathcal{L}_{q}^{h}(\eta)-\delta_{q}^{h}(\eta), \mathcal{L}_{q}^{h}(\eta)+\delta_{q}^{h}(\eta)\right]
$$

where

$$
\delta_{q}^{h}(\eta)=\left\|\mathcal{U}_{q}^{h}(\cdot, \eta) ; L_{2}\left(\omega_{+}^{h}\right)\right\|^{-1}\left\|\mathcal{A}_{q}^{h}(\eta) \mathcal{U}_{q}^{h}(\cdot, \eta)-\mathcal{L}_{q}^{h}(\eta) \mathcal{U}_{q}^{h}(\cdot, \eta) ; L_{2}\left(\omega_{+}^{h}\right)\right\|,
$$

contains at least one eigenvalue of the operator $\mathcal{A}_{q}^{h}(\eta)$.

In accordance with the asymptotic Ansatz (5.8), for the role of the "near eigenvalue" of Lemma 7.1 we take the sum

$$
\mathcal{L}_{q}^{h}(\eta)=h^{-2} \mu_{1}+e^{-m / h} \Lambda_{q}^{h \prime}(\eta)
$$


where $\Lambda_{q}^{h \prime}(\eta)$ is the quantity (5.17) if $q=1$, or (5.16) if $q=2$ (accordingly, (5.14) or (5.13) for $l=1)$. Observe that

$$
e^{-m / h}\left|\Lambda_{q}^{h \prime}(\eta)\right| \leq c\left(e^{-m / h}+e^{-m l / h}\right) .
$$

The "near eigenfunction" $\mathcal{U}_{q}^{h}(\cdot, \eta)$ looks bulkier:

$$
\begin{aligned}
\mathcal{U}_{q}^{h}(x, \eta) & =w_{1}(\xi)+e^{-m / h}\left(a_{q}(\eta) W_{1}(\xi)+b_{q}(\eta) W_{2}(\xi)+\Lambda_{q}^{h \prime}(\eta) W^{\prime}(\xi)\right) \\
& -\tilde{\chi}^{h}(x)\left(\widetilde{w}_{1}(\xi)+e^{-m / h}\left(a_{q}(\eta) \widetilde{W}_{1}(\xi)+b_{q}(\eta) \widetilde{W}_{2}(\xi)+\Lambda_{q}^{h \prime}(\eta) \widetilde{W}^{\prime}(\xi)\right)\right) .
\end{aligned}
$$

Here $\xi=\left(h^{-1}\left(x_{1}-l / 2\right), h^{-1} x_{2}\right)$ denotes the fast variables, the factors $a_{q}(\eta)$ and $b_{q}(\eta)$ are given by (5.5), and $\widetilde{w}_{1}$ and $\widetilde{W}_{1}, \widetilde{W}_{2}, \widetilde{W}^{\prime}$ are the remainder terms in (3.6) and (3.10). Also, $\tilde{\chi}^{h}$ is a smooth cut-off function equal to 1 in the $c h$-neighborhoods of the segments $I_{0}^{h}$ and $I_{ \pm}^{h}=\left\{x:\left|x_{1}-l / 2\right|<1 / 2, x_{2}= \pm 1 / 2\right\}$, but vanishing outside the 2 ch-neighborhoods.

By the theorem on asymptotics [21] and the weighted Hölder estimates [22] for solutions of elliptic boundary-value problems, in cylindrical domains we have

$$
\begin{aligned}
\left|\nabla_{\xi}^{j} \widetilde{w}_{1}(\xi)\right| \leq c_{j} \exp \left(-|\xi| \sqrt{4 \pi^{2}-\mu_{1}}\right), \\
\left|\nabla_{\xi}^{j} \widetilde{W}_{p}(\xi)\right| \leq c_{j} \exp \left(-|\xi| \sqrt{\pi^{2}-\mu_{1}}\right), \\
\left|\nabla_{\xi}^{j} \widetilde{W}^{\prime}(\xi)\right| \leq c_{j}|\xi| \exp \left(-|\xi| \sqrt{\pi^{2}-\mu_{1}}\right),
\end{aligned}
$$

where $\xi \in \overline{\Xi^{1}},|\xi| \geq \max \{1, l\}$ and $\nabla_{\xi}^{j} \widetilde{w}_{1}$ is the collection of the derivatives of $\widetilde{w}_{1}$ of order $j=0,1,2, \ldots$.

Remark 7.1. The coefficients of $|\xi|$ in the exponents in (7.4) are the same as for the particular solutions (3.5). We explain the origin of the additional factor $|\xi|$ in the last majorant. Since $W^{\prime}$ solves the Dirichlet problem for the nonhomogeneous Helmholtz equation (5.9) with the right-hand side $w_{1}(\xi)=O(\exp (-m|\xi|)$ that decays exponentially, the general theory of boundary-value problems in domains with cylindrical outlets to infinity shows that the growing terms of the expansion (3.10) remain the same as those for the solutions $W_{p}$ of the homogeneous problem (of course, the coefficients may be different). The remainder term $\widetilde{W}^{\prime}$ still belongs to the space $\mathcal{W}_{\beta}^{1}\left(\Xi^{1}\right)$ for any $\beta<m$, but the next terms, which vanish at infinity, differ from the corresponding terms for $W_{p}$ (the latter terms involve the particular solutions (3.5) occurring in (3.6), and this fact is reflected in the middle inequality in (7.4) ). The exponentially small terms for $W^{\prime}$ are "distorted" by the right-hand side $w_{1}$ of the Helmholtz equation, namely, by the theorem on asymptotics [21] (see, e.g., [6, Lemma 3.3.1]), the exponentials $e^{m \xi_{1}}$ and $e^{ \pm m \xi_{2}}$ (see (3.6) $)$ are replaced by the products $\Psi_{0}\left(\xi_{1}\right) e^{m \xi_{1}}$ and $\Psi_{ \pm}\left(\xi_{2}\right) e^{ \pm m \xi_{2}}$, where $\Psi_{0}$ and $\Psi_{ \pm}$are some linear functions; precisely this gives rise to the "extra" factor $|\xi|$ in the last line in (7.4).

Since the cut-off function $\tilde{\chi}^{h}$ occurs in (17.3), the quantity $\mathcal{U}_{q}^{h}(x, \eta)$ coincides near the segment mentioned above with the asymptotic terms analyzed in $\S 5$, i.e., with a linear combination of the leading terms of representations (3.6) and (3.10) for $w_{1}, W_{p}, W^{\prime}$ (e.g., near $I_{0}^{h}$ this is the product $\cos \left(\pi x_{2} / h\right)(\ldots)$, see (5.15)). Thus, by construction, the function $\mathcal{U}_{q}^{h}(\cdot, \eta)$ is subject to the required Dirichlet and quasiperiodicity conditions.

The fast decay of the remainder terms $\widetilde{w}_{1}$ and $\widetilde{W}_{q}, \widetilde{W}^{\prime}$, shown by (17.4), is of great importance: for this reason the discrepancies due to multiplication by a cut-off function turn out to be acceptably small (see the deduction of formula (7.11) below).

We calculate the norms occurring in (7.1). It is easy to verify the two-sided estimate

$$
C h^{2} \geq\left\|w_{1}-\tilde{\chi}^{h} \widetilde{w}_{1} ; L_{2}\left(\omega_{+}^{h}\right)\right\|^{2} \geq c h^{2}, \quad c>0
$$


where the factor $h^{2}$ comes from the coordinate dilation $x \mapsto \xi$. Applying the second inequality in (7.4) and recalling (3.10) and (3.18), we obtain

$$
\begin{aligned}
& e^{-2 m / h}\left\|W_{q}-\tilde{\chi}^{h} \widetilde{W}_{q} ; L_{2}\left(\omega_{+}^{h}\right)\right\|^{2} \\
& \quad \leq c h e^{-2 m / h}\left(h+\int_{h / 2}^{1 / 2} e^{2 m x_{2} / h} d x_{2}+\int_{-l / 2}^{h / 2} e^{-2 m x_{1} / h} d x_{1}\right) \\
& \quad \leq \operatorname{ch}^{2} e^{-2 m / h}\left(1+e^{m / h}+e^{m l / h}\right) .
\end{aligned}
$$

Condition (5.10) implies that the function $W^{\prime}$ decays exponentially in the half-strips $\Pi_{ \pm}^{1}$. Thus, by (7.2), we get

$$
\begin{aligned}
& e^{-2 m / h}\left|\Lambda_{q}^{h \prime}(\eta)\right|^{2}\left\|W^{\prime}-\tilde{\chi}^{h} \widetilde{W}^{\prime} ; L_{2}\left(\omega_{+}^{h}\right)\right\|^{2} \\
& \quad \leq \operatorname{ch}\left(e^{-m / h}+e^{-m l / h}\right)^{2}\left(h+\int_{-l / 2}^{h / 2} e^{-2 m x_{1} / h} d x_{1}\right) \\
& \quad \leq \operatorname{ch}^{2}\left(e^{-2 m / h}+e^{-2 m l / h}\right)\left(1+e^{m l / h}\right) .
\end{aligned}
$$

The above inequalities establish the upper estimate in the relation

$$
C h^{2}\left(1+e^{m(l-2) / h}\right) \geq\left\|\mathcal{U}_{q}^{h}(\cdot ; \eta) ; L_{2}\left(\omega_{+}^{h}\right)\right\|^{2} \geq c h^{2}\left(1+e^{m(l-2) / h}\right), \quad c>0 .
$$

To verify the lower one, for $l \leq 2$ we use the simple inequality

$$
\left\|\mathcal{U}_{q}^{h}(\cdot ; \eta) ; L_{2}\left(\omega_{+}^{h}\right)\right\|^{2} \geq\left\|\mathcal{U}_{q}^{h}(\cdot ; \eta) ; L_{2}\left(Q_{+}^{h}\right)\right\|^{2} \geq c h^{2}, \quad c>0
$$

(cf. the left inequality in (7.5)), which is valid due to the small coefficients of $W_{p}$, $W^{\prime} \in H^{1}\left(Q^{1}\right)$ in the definition (7.3). If $l>2$, then the factors $e^{-m / h}$ and $e^{-m / h} \lambda_{q}^{h^{\prime}}(\eta)$ have one and the same smallness order, but, since the functions $W_{2}$ and $W^{\prime}$ are linearly independent, while $w_{1}(\xi)$ decays rapidly in the half-strip $\Pi_{0}^{1}$, we can write

$$
\begin{aligned}
& \left\|\mathcal{U}_{q}^{h}(\cdot ; \eta) ; L_{2}\left(\omega_{+}^{h}\right)\right\|^{2} \geq\left\|\mathcal{U}_{q}^{h}(\cdot ; \eta) ; L_{2}\left(\omega_{+}^{h} \cap \varpi_{0}^{h}\right)\right\|^{2} \\
& \quad \geq \operatorname{ch} e^{-2 m / h}\left(\int_{-l / 2}^{-h / 2} e^{2 m x_{1} / h} d x_{1}-h\left(1+e^{m / h}\right)\right) \geq \operatorname{ch}^{2} e^{m(l-2) / h}, \quad c>0 .
\end{aligned}
$$

We continue the calculation of the quantity (7.1). The equations satisfied by $w_{1}, W_{p}$, and $W^{\prime}$ show that

$$
\begin{aligned}
& \Delta_{x} \mathcal{U}_{q}^{h}(x ; \eta)+\mathcal{L}_{q}^{h}(\eta) \mathcal{U}_{q}^{h}(x ; \eta)=e^{-m / h} \Lambda_{q}^{h \prime}(\eta)\left(\mathcal{U}_{q}^{h}(x ; \eta)-w_{1}(\xi)\right) \\
& \quad+\left[\Delta_{x}, \widetilde{\chi}^{h}\right]\left(\widetilde{w}_{1}(\xi)+e^{-m / h}\left(a_{q}(\eta) \widetilde{W}_{1}(\xi)+b_{q}(\eta) \widetilde{W}_{2}(\xi)+\lambda_{q}^{h \prime}(\eta) \widetilde{W}^{\prime}(\xi)\right)\right) .
\end{aligned}
$$

Here $\left[\Delta_{x}, \tilde{\chi}^{h}\right]$ is the commutator of the Laplace operator with the cut-off function,

$$
\left[\Delta_{x}, \tilde{\chi}^{h}\right] v=2 \partial_{1} v \partial_{1} \tilde{\chi}^{h}+2 \partial_{2} v \partial_{2} \tilde{\chi}^{h}+v \Delta_{x} \tilde{\chi}^{h},
$$

and the coefficients of this differential operator are nonzero only on the union of the $2 h$-neighborhood of the segments $I_{0}^{h}$ and $I_{ \pm}^{h}$; we denote this union by $\mathcal{O}^{h}$. Two facts should be mentioned. First, the functions $w_{1}$ and $W_{p}$ are solutions of the homogeneous Helmholtz equation, and $W^{\prime}$ solves the nonhomogeneous equation (precisely for this reason the coefficient of $e^{-m / h} \Lambda_{q}^{h \prime}(\eta)$ in (7.10) acquired the subtrahend $w_{1}=-\Delta_{\xi} W^{\prime}-$ $\left.\mu_{1} W^{\prime}\right)$. Second, the remainders $\widetilde{w}_{1}, \widetilde{W}_{p}$, and $\widetilde{W}^{\prime}$ satisfy the same equations as the corresponding functions themselves, and in the equation for $\widetilde{W}^{\prime}$ we have no need to put tilde above the right-hand side (precisely for this reason the coefficient of $e^{-m / h} \Lambda_{q}^{h \prime}(\eta)$ mentioned above does not involve the summand $\left.\tilde{\chi}^{h}(\xi) \widetilde{w}_{1}(\xi)\right)$. 
Consider the right-hand side of (7.10). The $L_{2}\left(\omega_{+}^{h}\right)$-norm of the term with the commutator does not exceed

$c h^{-2} h\left(e^{-\min \{1, l\} \sqrt{4 \pi^{2}-\mu_{1}} /(2 h)}+\left(e^{-m / h}+e^{-m l / h}\right)\left(1+h^{-\min \{1, l\}}\right) e^{-\min \{1, l\} \sqrt{\pi^{2}-\mu_{1}} /(2 h)}\right)$.

The factor $h^{-2}$ has arisen because of the double differentiation of the rapidly changing cut-off function $\tilde{\chi}^{h}$, the factor $h$ replaces the quantity $\left(\operatorname{mes}_{2} \mathcal{O}^{h}\right)^{1 / 2}$ (the area of the support of $\tilde{\chi}^{h}$ is $O\left(h^{2}\right)$ ), and the exponentials came as a result of applying (7.4) and (7.2). Finally, the expression $h^{-\min \{1, l\} / 2}$ originates from the coefficient $|\xi|$ in (17.4). Thus, we have found a majorant of the form $c N_{2}^{h}(l)$, where

$$
N_{2}^{h}(l)=h^{-2}\left(e^{-\min \{1, l\} \sqrt{4 \pi^{2}-\mu_{1}} / h}+h^{-2 \min \{1, l\}} e^{-3 m \min \{1, l\} / h}\right),
$$

for the square of the $L_{2}\left(\omega_{+}^{h}\right)$-norm of the second (with the commutator) summand on the right in (7.10). The same norm of the first summand can be handled with the help of similar tricks. Observe that

$$
\begin{gathered}
\left\|\mathcal{U}_{q}^{h}-w_{1} ; L_{2}\left(\omega_{+}^{h}\right)\right\|^{2} \leq 2 e^{-2 m / h}\left\|a_{q}(\eta) W_{1}+b_{q}(\eta) W_{2}+\Lambda_{q}^{h \prime}(\eta) W^{\prime} ; L_{2}\left(\omega_{+}^{h}\right)\right\|^{2} \\
+2\left\|\tilde{\chi}^{h}(\ldots) ; L_{2}\left(\omega_{+}^{h}\right)\right\|^{2},
\end{gathered}
$$

where the dots denote the factor of $\tilde{\chi}^{h}$ in (7.3). The second term on the right in (7.12) is dominated by the expression (7.11) (even with $h^{2}$ in place of $h^{-2}$, because we do not need to differentiate the cut-off function). The majorant $c N_{1}^{h}(l)$ for the first term, with

$$
N_{1}^{h}(l)=h^{2}\left(e^{-m / h}+e^{-m l / h}\right)^{2}\left(e^{-2 m / h} e^{m \max \{1, l\} / h}+\left(e^{-m / h}+e^{-m l / h}\right)^{2} e^{m l / h}\right),
$$

can be found with the help of (7.6) and (17.7).

Since

$$
\left\|\Delta_{x} \mathcal{U}_{q}^{h}+\mathcal{L}_{q}^{h}(\eta) \mathcal{U}_{q}^{h} ; L_{2}\left(\omega_{+}^{h}\right)\right\|=\sup \left|\left(\Delta_{x} \mathcal{U}_{q}^{h}+\mathcal{L}_{q}^{h}(\eta) \mathcal{U}_{q}^{h}, \mathcal{V}\right)_{\omega_{+}^{h}}\right|
$$

where the supremum is over all functions $\mathcal{V} \in H^{1}\left(\omega_{+}^{h}\right)$ with unit $L_{2}\left(\omega_{+}^{h}\right)$-norm and satisfying the required conditions on the boundary $\partial \omega_{+}^{h}$, we obtain the following estimate for the quantity (7.1):

$$
\delta_{q}^{h}(\eta) \leq c\left(1+e^{m(l-2) / h}\right)^{-1 / 2} h^{-1}\left(N_{1}^{h}(l)+N_{2}^{h}(l)\right)^{1 / 2} .
$$

Here, the first factor came from inequality (7.8), and the $N_{p}^{h}(l)$ are defined in (7.13) and (7.11).

Theorem 7.1. For the first two eigenvalues in the spectrum (1.10) of the model problem (1.5)-(1.8) (or (4.1) in the variational setting), we have the asymptotic representations (5.8), in which $\mu_{1} \in\left(0, \pi^{2}\right)$ is the eigenvalue of problem (2.1) in the domain $\Xi^{1}$ (see Figure 4 and Theorem 2.1), the asymptotic corrections $\Lambda_{q}^{h \prime}(\eta)$ have the form (5.17) and (5.16) (or (5.14) and (5.13) if $l=1$ ), and the remainder term (6.1) admits the estimate

$$
\left|\widetilde{\Lambda}_{q}^{h}(\eta)\right| \leq c \begin{cases}e^{-m / h,} & l \geq 2, \\ h^{-\varkappa} e^{-m / h} e^{-m \alpha(l) /(2 h)}, & l \in[1,2), \\ h^{-\varkappa} e^{-m l / h} e^{-m \alpha(l) l /(2 h)}, & l \in(0,1),\end{cases}
$$

where $\varkappa$ is some exponent, and $\alpha(l)$ is the positive quantity defined for $l<2$ by

$$
\alpha(l)=\min \left\{1,|2-l|, \frac{\sqrt{4 \pi^{2}-\mu_{1}}}{\sqrt{\pi^{2}-\mu_{1}}}-2\right\} .
$$


Of course, the deduction of estimate (7.15) has required only simplified majorants (7.11) and (7.13), because to justify the claims of Theorem6.1 it suffices to have the positive supplement (7.16) to the exponent only in the second and third lines of (7.15). By the way, $\alpha(l)>0$ because $\sqrt{4 \pi^{2}-\mu_{1}}>2 \sqrt{\pi^{2}-\mu_{1}}$. If $l \geq 2$, then by (7.8) and (7.13), (7.11), we have

$$
\begin{aligned}
& \left\|\mathcal{U}_{q}^{h} ; L_{2}\left(\omega_{+}^{h}\right)\right\|^{-2} \leq c h^{-2} e^{-m(l-2) / h}, \quad N_{1}^{h}(l) \leq h^{2} e^{-2 m / h} e^{m(l-2) / h}, \\
& N_{2}^{h}(l) \leq h^{-2}\left(e^{-l \sqrt{4 \pi^{2}-\mu_{1}} / h}+h^{-2 l} e^{-3 m l / h}\right) \leq c h^{2} e^{-2 m / h} e^{m(l-2) / h} .
\end{aligned}
$$

In the above estimate for $N_{2}^{h}(l)$ we used the fact that $e^{-l \sqrt{4 \pi^{2}-\mu_{1}} / h} \leq e^{-4 m / h}$ and $h^{-N} e^{-n / h} \leq C$ for any $h \in(0,1), n>0$, and $N$. Moreover, the same inequalities allow us to drop the power factor $h^{-\varkappa}$ in formula (6.4) of Theorem 6.1(1). The first two relations (7.17) show that on the basis of the results obtained it is impossible to refine the estimate of the asymptotic remainder (6.1) for $l \geq 2$.

\section{§8. Discrete spectrum}

We introduce a domain $\Omega_{\natural}^{h}$ as a perturbation of the domain (1.1): one of the treads, say $s_{0}^{h}$ (see (1.13) with $p=0$ ), will be replaced with a tread $s_{0}^{h H}$ of thickness $h H$ :

$$
s_{0}^{h H}=\left\{x:\left|x_{1}\right|<l / 2,\left|x_{2}\right|<h H / 2\right\} .
$$

Here $H \in\left(0, H_{*}\right)$ is a parameter, which may be assumed to differ little from 1 (recall that the proof of Theorem [2.1. as presented in $\S 2$, involves $\left.H \in\left(1-\delta_{-}^{H}, 1+\delta_{+}^{H}\right)\right)$. Like in $\S 1$, we introduce the positive selfadjoint operator $A_{\natural}^{h}$ in the space $L_{2}\left(\Omega_{\natural}^{h}\right)$ that corresponds (see [2, § 10.1]) to the variational setting of problem (1.2) in $\Omega_{\natural}^{h}$ :

$$
\left(\nabla_{x} u_{\natural}^{h}, \nabla_{x} v_{\natural}^{h}\right)_{\Omega_{\natural}^{h}}=\lambda_{\natural}^{h}\left(u_{\natural}^{h}, v_{\natural}^{h}\right)_{\Omega_{\natural}^{h}}, \quad v_{\natural}^{h} \in \stackrel{\circ}{H}^{1}\left(\Omega_{\natural}^{h}\right) .
$$

Since the domain perturbation occurs inside a bounded domain, the operator $A_{\natural}^{h}$ still has the set (1.12) as its essential spectrum. We shall show that, unlike the case of the operator $A^{h}$, for which $\sigma_{d}\left(A^{h}\right)=\varnothing$, the discrete spectrum of $A_{\natural}^{h}$ is not empty.

First, let $H \in\left(1, H_{*}\right)$, i.e., the tread (8.1) is thicker than the other treads. By Theorems 2.1 and 2.2, the operator $B^{H}$ of problem (2.4) has an eigenvalue

$$
\mu_{H} \in\left(0, \mu_{1}\right) .
$$

The corresponding eigenfunction $w_{H} \in \stackrel{\circ}{H}^{1}\left(\Omega^{H}\right)$ satisfies the estimates

$$
\begin{aligned}
& \left.\mid w_{H}(\xi)\right)|+| \nabla_{\xi} w_{H}(\xi) \mid \leq c \exp \left(-\left|\xi_{2}\right| \sqrt{\pi^{2}-\mu_{H}}\right), \quad \xi \in \Pi_{ \pm}^{H}, \pm \xi_{2}>1+H / 2, \\
& \left.\mid w_{H}(\xi)\right)|+| \nabla_{\xi} w_{H}(\xi) \mid \leq c \exp \left(\xi_{1} \sqrt{H^{-2} \pi^{2}-\mu_{H}}\right), \quad \xi \in \Pi_{0}^{H}, \pm \xi_{1}<-1 .
\end{aligned}
$$

We put $m_{H}=\sqrt{H^{-2} \pi^{2}-\mu_{H}}$ and observe that $m_{H}<\sqrt{\pi^{2}-\mu_{H}}$. By analogy with (4.3), we introduce the test functions

$$
U_{ \pm}^{h}(x)=\chi_{ \pm}(x) w_{H}\left( \pm h^{-1}\left(x_{1} \mp l / 2\right), h^{-1} x_{2}\right) .
$$

Repeating the calculations (4.4), (4.5) and using (8.2), we get

$$
\begin{aligned}
c_{0} \exp \left(-\frac{2}{3} \frac{\rho}{h} m_{H}\right) & \leq\left|\left\|U_{ \pm}^{h} ; L_{2}\left(\Omega_{\natural}^{h}\right)\right\|^{2}-h^{2}\left\|w_{H} ; L_{2}\left(\Xi^{H}\right)\right\|^{2}\right| \\
& \leq C_{0} \exp \left(-\frac{2}{3} \frac{\rho}{h} m_{H}\right), \quad c_{0}>0, \\
\mid\left\|\nabla_{x} U_{ \pm}^{h} ; L_{2}\left(\Omega_{\natural}^{h}\right)\right\|^{2} & -\left\|\nabla_{\xi} w_{H} ; L_{2}\left(\Xi^{H}\right)\right\|^{2} \mid \leq C_{1} \exp \left(-\frac{2}{3} \frac{\rho}{h} m_{H}\right) .
\end{aligned}
$$

Since

$$
\left\|\nabla_{\xi} w_{H} ; L_{2}\left(\Xi^{H}\right)\right\|^{2}=\mu_{H}\left\|w_{H} ; L_{2}\left(\Xi^{H}\right)\right\|^{2}
$$


(cf. (2.4) ), we can conclude that

$$
\begin{aligned}
\frac{\left\|\nabla_{x} U_{ \pm}^{h} ; L_{2}\left(\Omega_{\natural}^{h}\right)\right\|^{2}}{\left\|U_{ \pm}^{h} ; L_{2}\left(\Omega_{\natural}^{h}\right)\right\|^{2}} & \leq \frac{1}{h^{2}} \frac{\left\|\nabla_{\xi} w_{H} ; L_{2}\left(\Xi^{H}\right)\right\|^{2}+c \exp \left(-2 h^{-1} m_{H} /(3 \rho)\right)}{\left\|w_{H} ; L_{2}\left(\Xi^{H}\right)\right\|^{2}-c \exp \left(-2 h^{-1} m_{H} /(3 \rho)\right)} \\
& \leq \frac{1}{h^{2}}\left(\mu_{H}+C \exp \left(-\frac{2}{3} \frac{\rho}{h} m_{H}\right)\right) .
\end{aligned}
$$

If $h$ is small, then the right-hand side of (8.5) falls within the interval $\left(0, h^{-2}\left(\mu_{1}-\delta_{1}\right)\right)$ which, by Theorem 6.1(1), is free of the essential spectrum of $A_{\natural}^{h}$ for any $\delta_{1}>0$. Then Theorem 10.2.2 in 2] (the maximin principle) says that there are at least two (by the number of linearly independent test functions in (8.3)) eigenvalues of $A_{\natural}^{h}$. By increasing the number of thickened treads, we can place any prescribed number of eigenvalues on the interval $\left(0, h^{-2}\left(\mu_{1}-\delta_{1}\right)\right)$ located below the essential spectrum (1.12).

Now, let $H<1$. Formulas (8.3) and (8.5) remain valid, but Theorem 10.2.2 of [2] is no longer applicable, because, by Theorem 6.1(1), the part $\Upsilon_{1}^{h} \cup \Upsilon_{2}^{h}$ of the essential spectrum (1.12) of $A_{\natural}^{h}$ is located below the point $h^{-2} \mu_{H}$. We refer to the spectral measure theory (see, e.g., 2, Chapter 5 and 6]). By the spectral theorem (see [2, Theorem 6.1.1]), the selfadjoint operator $A_{\natural}^{h}$ in $L_{2}\left(\Omega_{\natural}^{h}\right)$ gives rise to a spectral measure $2 \mathbf{E}_{A_{\natural}^{h}}$, which, in its turn, associates the scalar complex measure $\boldsymbol{\mu}_{\mathbf{u}^{h}, \mathbf{v}^{h}}=\left(\mathbf{E}_{A_{\natural}^{h}} \mathbf{u}^{h}, \mathbf{v}^{h}\right)_{\Omega_{\natural}^{h}}$ on $\mathbb{R}$ with each pair $\mathbf{u}^{h}, \mathbf{v}^{h} \in L_{2}\left(\Omega_{\natural}^{h}\right)$. We shall need the following simple formulas (see, e.g., the proof of Theorem 6.1.3 in [2]):

$$
\left(\mathbf{u}^{h}, \mathbf{v}^{h}\right)_{\Omega_{\natural}^{h}}=\int_{\mathbb{R}} d \boldsymbol{\mu}_{\mathbf{u}^{h}, \mathbf{v}^{h}}(\mathbf{t})
$$

and, for $\boldsymbol{\lambda}^{h} \in \mathbb{R}$ and $\mathbf{u}^{h}$ in the domain $\mathcal{D}\left(A_{\natural}^{h}\right)$ of the operator $A_{\natural}^{h}$,

$$
\left\|A_{\natural}^{h} \mathbf{u}^{h}-\boldsymbol{\lambda}^{h} \mathbf{u}^{h} ; L_{2}\left(\Omega_{\natural}^{h}\right)\right\|^{2}=\int_{\mathbb{R}}\left(\mathbf{t}-\boldsymbol{\lambda}^{h}\right)^{2} d \boldsymbol{\mu}_{\mathbf{u}^{h}, \mathbf{u}}^{h}(\mathbf{t}) .
$$

Let

$$
\boldsymbol{\lambda}^{h}=h^{-2} \mu_{H} \in\left(h^{-2} \mu_{1}, h^{-2} \pi^{2}\right)
$$

(recall that $H<1$ ). By Theorems 4.1 and 6.1(1), in the $c h^{-2}$-neighborhood of the point $\lambda^{h}$ the measure $\mathbf{E}_{A^{h}}$ is discrete (a linear combination of Dirac functions). Suppose that, for some $\ell>0$, the interval

$$
\Sigma=\left(\frac{\mu_{H}}{h^{2}}-\ell h^{-1 / 2} \exp \left(-\frac{m_{H}}{3 h}\right), \frac{\mu_{H}}{h^{2}}+\ell h^{-1 / 2} \exp \left(-\frac{m_{H}}{3 h}\right)\right)
$$

is free of the eigenvalues of $A_{\natural}^{h}$, i.e., $\mathbf{E}_{A_{\natural}^{h}}(\Sigma)=0$. Then

$$
\begin{aligned}
& \left\|A_{\natural}^{h} U_{ \pm}^{h}-h^{-2} \mu_{H} U_{ \pm}^{h} ; L_{2}\left(\Omega_{\natural}^{h}\right)\right\|^{2} \\
& \quad=\int_{\mathbb{R}}\left(\mathbf{t}-h^{-2} \mu_{h}\right)^{2} d \boldsymbol{\mu}_{U_{ \pm}^{h}, U_{ \pm}^{h}}(\mathbf{t}) \geq c \ell^{2} h^{-1} \exp \left(-\frac{2 m_{H}}{3 h}\right) \int_{\mathbb{R}} d \boldsymbol{\mu}_{U_{ \pm}^{h}, U_{ \pm}^{h}} d(\mathbf{t}) \\
& \quad=c \ell^{2} h^{-1} \exp \left(-\frac{2 m_{H}}{3 h}\right)\left\|U_{ \pm}^{h} ; L_{2}\left(\Omega_{\natural}^{h}\right)\right\|^{2} .
\end{aligned}
$$

At the same time, we have

$$
\Delta_{x} U_{ \pm}^{h}(x)+h^{-2} \mu_{H} U_{ \pm}^{h}(x)=\chi_{ \pm}(x)\left(\Delta_{x} w_{H}(\xi)+h^{-2} \mu_{H} w_{H}(\xi)\right)+\left[\Delta_{x}, \chi_{ \pm}(x)\right] w_{H}(\xi)
$$

and the first term on the right is zero; therefore,

$$
\left\|\Delta_{x} U_{ \pm}^{h}+h^{-2} \mu_{H} U_{ \pm}^{h} ; L_{2}\left(\Omega_{\natural}^{h}\right)\right\|^{2} \leq c h \exp \left(-\frac{2 m_{H}}{3 h}\right) .
$$

\footnotetext{
${ }^{2}$ For the reader's convenience, we keep the notation of [2], but write it in bold letters.
} 
Comparing (8.8) and (8.4), we see that inequality (8.7) becomes absurd if $\ell$ is large. This contradiction establishes the existence of an eigenvalue on the interval (8.6) contained in the gap $G_{23}^{h}$. Actually, we have at least two eigenvalues there, because we can use the trick of posing artificial boundary conditions of the form (5.1) on the interval $I_{h}^{0}$.

Theorem 8.1. Let numbers $H_{ \pm 1}, \ldots, H_{ \pm N_{ \pm}}$be given, with

$$
1-\delta_{-}^{h}<H_{-N_{-}}<\cdots<H_{-1}<1<H_{1}<\cdots<H_{N_{+}}<1+\delta_{+}^{h} .
$$

Suppose that the domain $\Omega_{\natural}^{h}$ is obtained from the domain (1.1) by replacement of the treads (1.13) with the treads $s_{p}^{h H_{p}}$ (cf. (8.1)), where $p=p_{-N_{-}}, \ldots, p_{N_{+}}$and $p_{j} \neq p_{k}$ if $j \neq k$. Then for any small $h>0$, the discrete spectrum of the operator $A_{\natural}^{h}$ has at least $2 N_{+}$distinct eigenvalues below the essential spectrum (1.12) and at least $2 N_{-}$distinct eigenvalues inside the gap $G_{23}^{h}$.

The fact that the eigenvalues found in Theorem 8.1 are pairwise distinct follows from the strict inequalities in (8.9), the monotonicity of the function $H \mapsto \mu_{H}$, proved in Theorem 2.2. and the possibility to apply artificial boundary conditions like (5.1). Of course, the requirement $H_{j} \neq H_{k}$ for $j \neq k$ can be reduced, but then in the theorem we shall talk of the multiplicity of the discrete spectrum. The limits $1-\delta_{-}^{h}$ and $1+\delta_{+}^{h}$ are posed in (8.9) with the purpose that only the part of Theorem 2.1 that was verified in $\S 3$ be employed. If we refer to the full statement of Theorem 2.1, then we can start the list in (8.9) with zero and end with critical width $H_{*}$. By using the same tricks as in $\S 5$, it is not hard to construct the asymptotics of eigenvalues and check that there are no other discrete spectrum points on the interval $\left(0, h^{-2} \pi^{2}\right)$, but we do not dwell on this in the present paper.

\section{REFERENCES}

[1] O. A. Ladyzhenskaya, Boundary value problems of mathematical physics, Nauka, Moscow, 1973; English transl., Appl. Math. Sci., vol. 49, Springer-Verlag, New York, 1985. MR 0599579 (58:29032) MR0793735 (87f:35001)

[2] M. Sh. Birman and M. Z. Solomyak, Spectral theory of selfadjoint operators in Hilbert space, Leningrad. Univ., Leningrad, 1980; English transl., D. Reidel Publ. Co., Dordrecht, 1987. MR0609148 (82k:47001) MR1192782 (93g:47001)

[3] I. M. Gel'fand, Expansion in characteristic functions of an equation with periodic coefficients, Dokl. Akad. Nauk SSSR 73 (1950), no. 6, 1117-1120. (Russian) MR.0039154 (12:503a)

[4] P. A. Kuchment, Floquet theory for partial differential equations, Uspekhi Mat. Nauk 37 (1982), no. 4, 3-52; English transl., Russian Math. Surveys 37 (1982), no. 4, 60-94. MR0667973(84b:35018)

[5] S. A. Nazarov, Properties of spectra of boundary value problems in cylindrical and quasicylindrical domain, Sobolev Spaces in Mathematics. II (V. Maz'ya, ed.), Int. Math. Ser. (N. Y.), vol. 9, Springer, New York, 2009, pp. 261-309. MR2484629 (2009m:35508)

[6] S. A. Nazarov and B. A. Plamenevskiu, Elliptic problems in domains with piecewise smooth boundaries, Nauka, Moscow, 1991; English transl., de Gruyter Exp. in Math., vol. 13, Walter de Gruyter and Co., Berlin, 1994. MR1283387 (95h:35001)

[7] P. Kuchment, Floquet theory for partial differential equations, Oper. Theory Adv. Appl., vol. 60, Birkhäuser, Basel, 1993. MR1232660 (94h:35002)

[8] T. Kato, Perturbatin theory for linear operators, Springer-Verlag, Berlin, $1995 . \quad$ MR 1335452 (96a:47025)

[9] S. A. Nazarov, Elliptic boundary value problems with periodic coefficients in a cylinder, Izv. Akad. Nauk SSSR Ser. Mat. 45 (1981), no. 1, 101-112; English transl. in Math. USSR-Izv. 18 (1982), no. 1. MR0607578(82e:35035)

[10] Y. Avishai, D. Bessis, B. G. Giraud, and G. Mantica, Quantum bound states in open geometries, Phys. Rev. B 44 (1991), no. 15, 8028-8034.

[11] P. Duclos and P. Exner, Curvature-induced bound states in quantum waveguides in two and three dimensions, Rev. Math. Phys. 7 (1995), no. 1, 73-102. MR.1310767 (95m:81239) 
[12] P. Exner, P. Šeba, M. Tater, and D. Vaněk, Bound states and scattering in quantum waveguides coupled laterally through a boundary window, J. Math. Phys. 37 (1996), no. 10, 4867-4887. MR1411612 (98b:81032)

[13] D. Borisov, P. Exner, R. Gadyl'shin, and D. Krejčiřik, Bound states in weakly deformed strips and layers, Ann. Henri Poincaré 2 (2001), 553-572. MR1846856 (2002f:35053)

[14] S. A. Nazarov, Discrete spectrum of cranked, branching, and periodic waveguides, Algebra i Analiz 23 (2011), no. 2, 206-247; English transl. in St. Petersburg Math. J. 23 (2012), no. 2. MR2841676 (2012f:35382)

[15] , Localized waves in T-shape conjugation waveguide, Akust. Zh. 57 (2010), no. 6, 747-758. (Russian)

[16] K. Joshitomi, Band gap in the spectrum of periodically curved quantum waveguides, J. Differential Equations 142 (1998), no. 1, 123-166. MR1492880 (99a:35038)

[17] L. Friedlander and M. Solomyak, On the spectrum of narrow periodic waveguides, Russ. J. Math. Phys. 15 (2008), no. 2, 238-242. MR2410832(2009b:35310)

[18] S. A. Nazarov, A gap in the essential spectrum of the Neumann problem for an elliptic system in a periodic domain, Funktsional. Anal. i Prilozhen. 43 (2009), no. 3, 92-95; English transl., Funct. Anal. Appl. 43 (2009), no. 3, 239-241. MR2583962 (2010m:35339)

[19] _ An example of multiple gaps in the spectrum of a periodic waveguide, Mat. Sb. 201 (2010), no. 4, 99-124; English transl., Sb. Math. 201 (2010), no. 3-4, 569-594. MR2675342 (2012c:35096)

[20] V. I. Smirnov, A course of higher mathematics. Vol. II, Nauka, Moscow, 1967; English transl., Pergamon Press, Oxford, 1964. MR0182687 (32:170)

[21] V. A. Kondrat'ev, Boundary value problems for elliptic equations in domains with conical or angular points, Trudy Moskov. Mat. Obshch. 16 (1967), 209-292; English transl. in Trans. Moscow Math. Soc. 1967 (1968). MR.0226187 (37:1777)

[22] V. G. Maz'ya and B. A. Plamenevskir, The coefficients in the asymptotics of solutions of elliptic boundary value problems with conical points, Math. Nachr. 76 (1977), 29-60. (Russian) MR.0601608 (58:29176)

[23] - Estimates in $L_{p}$ and in Hölder classes, and the Miranda-Agmon maximum principle for the solutions of elliptic boundary value problems in domains with singular points on the boundary, Math. Nachr. 81 (1978), 25-82. (Russian) MR.0492821 (58:11886)

[24] S. A. Nazarov, Polynomial property of selfadjoint elliptic boundary value problems, and the algebraic description of their attributes, Uspekhi Mat. Nauk 54 (1999), no. 5, 77-142; English transl., Russian Math. Surveys 54 (1999), no. 5, 947-1014. MR.1741662 (2001k:35073)

[25] - On the asymptotics by parameter of the solution to a boundary value problem with periodic coefficients in cylinder, Differents. Uravneniya i Primenen. (Vilnius) 30 (1981), 27-44. (Russian)

[26] W. G. Mazja, S. A. Nasarow, and B. A. Plamenewski, Asymptotische Theorie elliptischer Randwertaufgaben in singulär gestörten Gebieten. 1, Akademie-Verlag, Berlin, 1991. (English transl., Maz'ya V., Nazarov S., Plamenevskij B., Asymptotic theory of elliptic boundary value problems in singularly perturbed domains. Vol. 1, Birkhäuser, Basel, 2000). MR1101139 (92g:35059) MR1779977 (2001e:35044a)

[27] M. I. Vishik and L. A. Lyusternik, Regular degeneration and boundary layer for linear differential equations with small parameter, Uspekhi Mat. Nauk (N. S.) 12 (1957), no. 5, 3-122; English transl., Amer. Math. Soc. Transl. (2), vol. 20, Amer. Math. Soc., Providence, RI, 1962, pp. $239-364$. MR0096041(20:2539); MR0136861(25:322)

Institute of Mechanical Engineering Problems, Bol'shoŭ Pr. V.O. 61, 199178 St.Petersburg, RUSSIA

E-mail address: srgnazarov@yahoo.co.uk

Received 25/JAN/2010

Translated by A. I. PLOTKIN 